\title{
An analytically-based method for predicting the noise generated by the interaction between turbulence and a serrated leading edge
}

\author{
J. R. Mathews ${ }^{\mathrm{a}, *}$, N. Peake ${ }^{\mathrm{a}}$ \\ ${ }^{a}$ Department of Applied Mathematics and Theoretical Physics, University of Cambridge, Cambridge.
}

\begin{abstract}
This paper considers the interaction of turbulence with a serrated leading edge. We investigate the noise produced by an aerofoil moving through a turbulent perturbation to uniform flow by considering the scattered pressure from the leading edge. We model the aerofoil as an infinite half plane with a leading edge serration, and develop an analytical model using a Green's function based upon the work of Howe. This allows us to consider both deterministic eddies and synthetic turbulence interacting with the leading edge. We show that it is possible to reduce the noise by using a serrated leading edge compared with a straight edge, but the optimal noise-reducing choice of serration is hard to predict due to the complex interaction. We also consider the effect of angle of attack, and find that in general the serrations are less effective at higher angles of attack.
\end{abstract}

Keywords: Leading edge serration, synthetic turbulence, eddies, Green's function

\section{Introduction}

Demand for air travel is booming, and is leading to the expansion of airports and the creation of new routes, with the number of aircraft growing by around $4 \%$ a year. Flightpath 2050 [6] has set out a roadmap to the middle of the century with key environmental targets such as a $90 \%$ cut in nitrous oxide emissions, a $75 \%$ cut in carbon dioxide emissions and a $65 \%$ reduction in effective perceived noise, all in comparison with the 2000 levels. The largest contributor at present to the noise is the aircraft engine, although radical future designs may completely change the dominant noise sources.

A modern turbofan has many sources of noise, and one of the key sources is fan noise. Peake and Parry [24] identified several different components such as rotor (or fan) self-noise, rotor-stator (or fan-vane) interaction, rotor casing boundary-layer interaction and droop-fan interaction.

In this paper we will focus on the rotor-stator interaction noise, which has both broadband and tonal elements, and is one of the dominant broadband and tonal sources of noise. The stators (or outlet guide vanes) straighten the swirling flow, but they do so at the expense of creating noise. The rotor wake is the sum of a uniform rotating flow plus a turbulent wake from each rotor blade, with a typical wake evolution shown in Cooper and Peake [5, Figure 2]. The turbulent wake from the rotor then interacts with the stators that block the wake, producing broadband noise. There is also tonal noise produced at harmonics of the blade passing frequency. By tuning the turbofan with different combinations of rotor and stator blades we the tonal noise can be controlled $[25,28]$.

This study considers flow-blade interaction in the context of the turbulent wake of the rotor hitting a single aerofoil, a stator blade. Several analytical models have been developed for the interaction of turbulence with an aerofoil, such as the theories of Amiet [1] and Howe [11]. Both theories involve considering only the trailing edge noise from a semi-infinite aerofoil and using a Green's function of the half plane. Howe's

\footnotetext{
* Corresponding author

Email addresses: jrm214@cam.ac.uk (J. R. Mathews), np100@cam.ac.uk (N. Peake)
} 
model assumes that the flow is at a low Mach number and the turbulence is frozen. Amiet's model is valid for all subsonic mean flows, and differs from Howe's in how the turbulence interacts with the aerofoil. Howe extended his theory to both sinusoidal [12] and sawtooth [13] serrations, and showed that serrations reduce the noise. According to his theory, reducing the wavelength of the serrations (and hence making them less shallow) reduces the noise. However, his Green's function was only valid for shallow serrations. Howe also extended his theory to aerofoils with a finite chord [16]. Roger and Moreau [26] extended Amiet's model in two ways. Firstly, they considered three dimensions and secondly they included the effect of back-scattering. Recently, Lyu et al. [20] proposed a new method to consider trailing edge serrations, by generalising Amiet's model to sawtooth serrations.

A very recent paper by Lyu and Azarpeyvand [19] extended Amiet's leading edge noise-prediction model to leading edge sawtooth serrations. Their analytical model compared favourably to experiments, and the noise reduction techniques were examined, with the primary mechanism destructive interference. Using the extension of Amiet's model as in their paper also allows the directivity of the leading edge noise to be closely examined, while Howe's model does not consider the directivity of the noise.

There have been a number of recent experimental and numerical studies on the effect of the serrations. A study from Haeri et al. [10] showed numerically that leading edge serrations reduce aerofoil noise. Additionally, experimental work by Gruber [8], Gruber et al. [9] and Narayanan et al. [23] further validated the theory that leading edge serrations can reduce aerofoil noise by a significant amount. In particular, Gruber [8] showed that Howe's model over-predicts the sound reduction from serrations, due to the assumption of frozen turbulence. However, the Green's function from Howe's method is only valid for shallow serrations, which is not the case for the serrations in Gruber [8, Figure 4.4].

Recent studies by Chaitanya et al. [3, 4] have considered a variety of different leading edge serrations, such as double-wavelength serrations, and their effect on noise performance and aerodynamic performance experimentally. They showed that it is generally possible to reduce the noise without comprising too much on aerodynamic performance.

In this paper we use the Green's function from Howe's analytical model and the model of turbulence from Haeri et al. [10] to investigate the effect of turbulent flow interacting with an aerofoil with a sinusoidal serrated leading edge. We show it is possible to reduce the noise by using a serrated leading edge, but it is hard to predict the correct choice of serration to minimise the noise.

\subsection{Organisation}

This paper is laid out as follows. In Section 2 we review Howe's method for calculating the pressure from an aerofoil in unsteady flow, and in Section 3 we derive the necessary Green's function for a straight-edged aerofoil and a serrated aerofoil. In Section 4 we calculate the pressure from the synthetic turbulence in Haeri et al. [10]. In Section 5 we consider synthetic turbulence comprising of a single eddy, and consider the effect of the eddy and flow parameters on the noise. In Section 6 we consider synthetic turbulence comprising of multiple eddies, and again show that the amount of noise reduction and optimal geometry of serrations vary significantly with the properties of the turbulence. Finally, in Section 7 we consider an aerofoil at a small angle of attack, and show that the angle of attack generally reduces the effectiveness of the serrations.

In this paper, we consider for the first time synthetic turbulence generated by eddies in Howe's model. We calculate the scattered pressure analytically, although we need to calculate one integral numerically. It is also the first time that multiple eddies interacting with each other in a non-linear way have been studied analytically in this context.

\subsection{Geometry of the aerofoil}

We model a single aerofoil blade as an infinitely thin half plane, and introduce a serration function $\mathscr{F}(z)$ (such as a sinusoidal wave or sawtooth) on the leading edge of the aerofoil (Figure 1). Mathematically, the aerofoil is defined by

$$
\left\{(x, y, z) \in \mathbb{R}^{3} \mid z \in \mathbb{R}, \quad \mathscr{F}(z) \cos \alpha<x<\infty, \quad y=x \tan \alpha\right\},
$$

where $\alpha$ is the angle of attack of the aerofoil. We introduce serrated cylindrical coordinates of $\left(r^{\star}, \theta^{\star}, z^{\star}\right)$, 
(a)

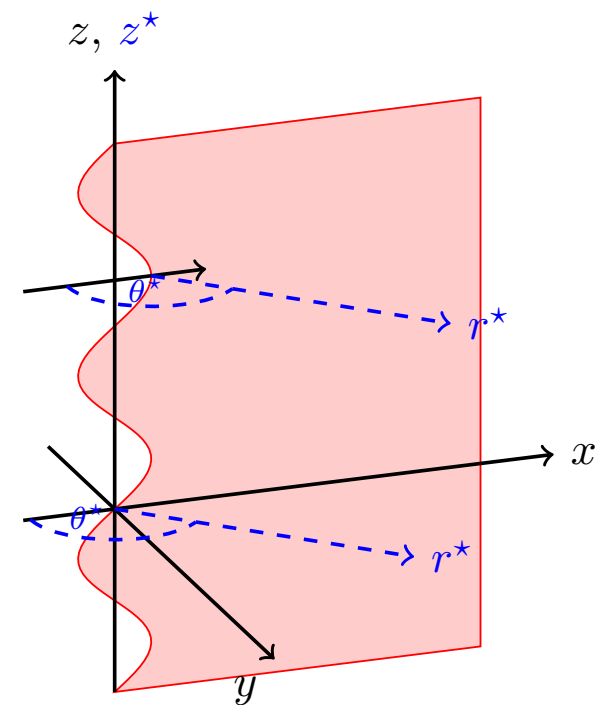

(b)

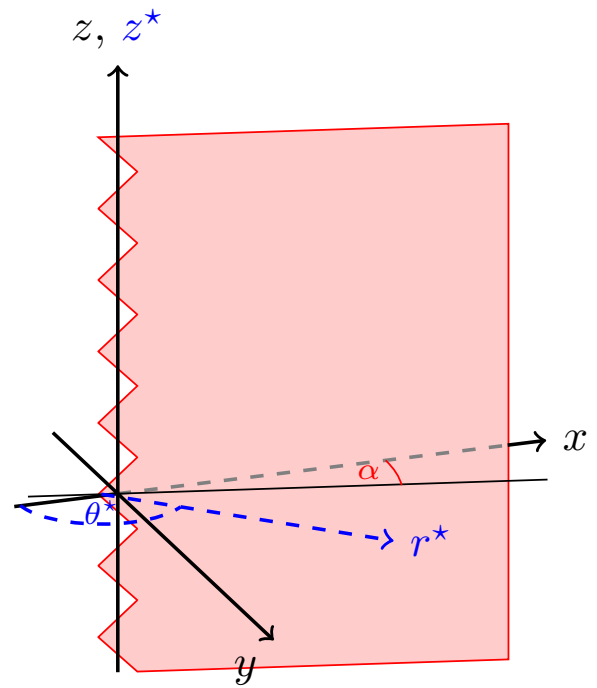

Figure 1: Geometry of serrated aerofoils in both Cartesian (black, solid) and serrated cylindrical coordinates (blue, dashed).

(a) Sinusoidal serrated aerofoil at zero angle of attack; (b) Sawtooth serrated aerofoil at angle of attack $\alpha$.

defined by

$$
(x, y, z)=\left(\mathscr{F}\left(z^{\star}\right) \cos \alpha-r^{\star} \cos \theta^{\star},-\mathscr{F}\left(z^{\star}\right) \sin \alpha-r^{\star} \sin \theta^{\star}, z^{\star}\right) .
$$

In these modified cylindrical coordinates $r^{\star}$ is the distance to the leading edge of the aerofoil, $\theta^{\star}$ is the angle in the $x-y$ plane, and $z^{\star}$ the height (Figure 1). Even at zero angle of attack, these coordinates are non-orthogonal, which will present difficulties in calculating the Laplacian later. We define $\theta^{\star}$ such that the two sides of the aerofoil correspond to $\theta^{\star}= \pm \pi-\alpha$.

When there is no angle of attack we calculate that

$$
(x, y, z)=\left(\mathscr{F}\left(z^{\star}\right)-r^{\star} \cos \theta^{\star},-r^{\star} \sin \theta^{\star}, z^{\star}\right),
$$

and hence the new coordinates are just cylindrical coordinates in the $x-y$ plane, centred at $\left(\mathscr{F}\left(z^{\star}\right), 0\right)$.

\subsubsection{Non-dimensionalisation}

We let $\ddagger$ represent all quantities with dimension. We non-dimensionalise all velocities by the (assumed constant) speed of sound $c_{0}^{\ddagger}$, so the non-dimensionalised speed of sound is given by $c_{0}=1$. Let the amplitude of the serration be $d^{\ddagger}$, and the dimensional frequency be $\omega^{\ddagger}$. We will non-dimensionalise all distances by $2 d^{\ddagger}$ to give a non-dimensionalised amplitude of 0.5 for all serrations, unless we are varying the amplitude in which case we don't have a fixed length scale. We calculate the non-dimensional frequency is given by

$$
\omega=\frac{2 \omega^{\ddagger} d^{\ddagger}}{c_{0}^{\ddagger}} .
$$

\section{Howe's approach}

We first make the assumption that the mean velocity flow is uniform and purely in the $x$ direction, i.e. is given by $\left(u_{0}, 0,0\right)$. We consider an aerofoil of the form in Figure 1, and non-dimensionalise as in Section 1.2.1. The assumption that the aerofoil is infinite is valid providing the chord of the aerofoil is sufficiently 
large relative to the acoustic wavelength of the turbulence hitting it. We follow the derivation in Howe [12] to calculate the pressure. We write the unsteady pressure $p(\boldsymbol{x}, t)$ as

$$
p(\boldsymbol{x}, t)=p_{i}(\boldsymbol{x}, t)+p_{s}(\boldsymbol{x}, t),
$$

where $p_{i}(\boldsymbol{x}, t)$ is the incident pressure field that would be present without the aerofoil. The scattered pressure $p_{s}(\boldsymbol{x}, t)$ is the extra pressure due to reflections and diffractions from the leading edge and the rest of the aerofoil.

Next, we introduce the Fourier transforms of pressure, $P_{i}(\boldsymbol{x}, \omega)$ and $P_{s}(\boldsymbol{x}, \omega)$, with

$$
p_{i}(\boldsymbol{x}, t)=\int_{\mathbb{R}} P_{i}(\boldsymbol{x}, \omega) e^{-\mathrm{i} \omega t} \mathrm{~d} \omega \text { and } p_{s}(\boldsymbol{x}, t)=\int_{\mathbb{R}} P_{s}(\boldsymbol{x}, \omega) e^{-\mathrm{i} \omega t} \mathrm{~d} \omega .
$$

For low Mach number flow, Howe [12] showed that

$$
\left(\Delta+\omega^{2}\right) P_{i}=S_{\text {source }}(\boldsymbol{x}, \omega) \text { and }\left(\Delta+\omega^{2}\right) P_{s}=0 .
$$

In Howe [12] the source term of the Helmholtz equation for the incident pressure is given explicitly, but is not needed here since we will only calculate the scattered pressure. We also have the boundary condition

$$
\frac{\partial P_{i}}{\partial y}+\frac{\partial P_{s}}{\partial y}=0
$$

on the aerofoil, corresponding to no-penetration across the rigid surface.

There are three ways described in Howe [15] to solve the scattering problem and find $P_{s}$. The first one involves the Weiner-Hopf procedure, the second involves Curle's representation of the pressure and the third uses the Kirchhoff integral and a Green's function. We choose the third method because it proved more convenient in this particular case.

The scattered pressure $P_{s}(\boldsymbol{x}, \omega)$ satisfies the Helmholtz equation everywhere. We introduce a Green's function $G\left(\boldsymbol{x} \mid \boldsymbol{x}_{\mathbf{0}} ; \omega\right)$ of the Helmholtz equation, satisfying

$$
\left(\Delta+\omega^{2}\right) G\left(\boldsymbol{x} \mid \boldsymbol{x}_{\mathbf{0}} ; \omega\right)=\delta\left(\boldsymbol{x}-\boldsymbol{x}_{\mathbf{0}}\right) .
$$

The Kirchhoff integral (essentially Green's second identity) then gives

$$
P_{s}(\boldsymbol{x}, \omega)=\oint\left(G\left(\boldsymbol{x} \mid \boldsymbol{x}_{\mathbf{0}} ; \omega\right) \frac{\partial P_{s}}{\partial \boldsymbol{n}}\left(\boldsymbol{x}_{\mathbf{0}}, \omega\right)-P_{s}\left(\boldsymbol{x}_{\mathbf{0}}, \omega\right) \frac{\partial G}{\partial \boldsymbol{n}}\left(\boldsymbol{x} \mid \boldsymbol{x}_{\mathbf{0}} ; \omega\right)\right) \mathrm{d} S\left(\boldsymbol{x}_{\mathbf{0}}\right),
$$

where $\boldsymbol{n}$ is the normal to the aerofoil pointing into the fluid, and the integration is on the surface of the aerofoil. We choose a tailored Green's function $G^{\mathscr{F}}$ of the Helmholtz equation, such that the normal derivative of the Green's function vanishes on the serrated aerofoil. We calculate this Green's function in Section 3. The scattered pressure is then given by

$$
\begin{aligned}
P_{s}(\boldsymbol{x}, \omega) & =\oint G^{\mathscr{F}}\left(\boldsymbol{x} \mid \boldsymbol{x}_{\mathbf{0}} ; \omega\right) \frac{\partial P_{s}}{\partial \boldsymbol{n}}\left(\boldsymbol{x}_{\mathbf{0}}, \omega\right) \mathrm{d} S\left(\boldsymbol{x}_{\mathbf{0}}\right), \\
& =-\oint G^{\mathscr{F}}\left(\boldsymbol{x} \mid \boldsymbol{x}_{\mathbf{0}} ; \omega\right) \frac{\partial P_{i}}{\partial \boldsymbol{n}}\left(\boldsymbol{x}_{\mathbf{0}}, \omega\right) \mathrm{d} S\left(\boldsymbol{x}_{\mathbf{0}}\right), \\
& =-\int_{-\infty}^{\infty} \int_{\mathscr{F}(x)}^{\infty} \frac{\partial P_{i}}{\partial y}\left(x_{0}, 0, z_{0}, \omega\right)\left[G^{\mathscr{F}}\left(\boldsymbol{x}, \boldsymbol{x}_{\mathbf{0}} ; \omega\right)\right] \mathrm{d} x_{0} \mathrm{~d} z_{0},
\end{aligned}
$$

where we have used (8), and where

$$
\left[G^{\mathscr{F}}\left(\boldsymbol{x}, \boldsymbol{x}_{\mathbf{0}} ; \omega\right)\right]=G^{\mathscr{F}}\left(\boldsymbol{x},\left(x_{0},+0, z_{0}\right) ; \omega\right)-G^{\mathscr{F}}\left(\boldsymbol{x},\left(x_{0},-0, z_{0}\right) ; \omega\right)
$$

is the jump of the Green's function across the aerofoil. We introduce a change of variables, $x_{1}=x_{0}+\mathscr{F}\left(z_{0}\right)$, to make the integration region simpler. This then gives

$$
P_{s}(\boldsymbol{x}, \omega)=-\int_{-\infty}^{\infty} \int_{0}^{\infty} \frac{\partial P_{i}}{\partial y}\left(x_{1}+\mathscr{F}(z), 0, z_{0}, \omega\right)\left[G^{\mathscr{F}}\left(\boldsymbol{x},\left(x_{1}+\mathscr{F}\left(z_{0}\right), 0, z_{0}\right) ; \omega\right)\right] \mathrm{d} x_{1} \mathrm{~d} z_{0} .
$$

Hence, once we have calculated the tailored Green's function, the scattered pressure can be found for any incident field. 


\section{Green's function for the Helmoltz equation tailored to an aerofoil with a serrated leading edge}

In this section we find the Green's function of the Helmholtz equation in (9), but with boundary conditions tailored to our aerofoil, so that the normal derivative in (10) vanishes on the aerofoil. We begin by calculating the exact Green's function for an infinite half plane.

\subsection{Infinite half plane}

The Green's function $G\left(\boldsymbol{x} \mid \boldsymbol{x}_{\mathbf{0}}\right)$ of the Helmholtz equation whose normal derivative vanishes on the aerofoil is given by

$$
\begin{aligned}
G\left(\boldsymbol{x} \mid \boldsymbol{x}_{\mathbf{0}}\right)=-\frac{\mathrm{i}}{8 \pi} \int_{-\infty}^{\infty} \sum_{m=0}\left[J_{\nu_{m}}(\gamma r) H_{\nu_{m}}^{(1)}\left(\gamma r_{0}\right) H\left(r_{0}-r\right)+\right. & \left.J_{\nu_{m}}\left(\gamma r_{0}\right) H_{\nu_{m}}^{(1)}(\gamma r) H\left(r-r_{0}\right)\right] \\
& \times a_{m} \cos \nu_{m}(\theta+\pi) \cos \nu_{m}\left(\theta_{0}+\pi\right) e^{\mathrm{i} k_{z}\left(z-z_{0}\right)} \mathrm{d} k_{z},
\end{aligned}
$$

where $\gamma\left(k_{z}\right)=\sqrt{\omega^{2}-k_{z}^{2}}, a_{0}=1, a_{m}=2$ for $m \geq 2, \nu_{m}=m / 2, J$ is the Bessel function, $H$ is the Heaviside function and $H^{(1)}$ is the Hankel function of the first kind. This agrees with the result in Howe [14] once we correct a typographical error and account for the slightly different geometries of the aerofoil. To derive the Green's function we take a Fourier series in $\theta$ and a Fourier transform in $z$ (with wavenumber $k_{z}$ ), which then only requires finding the Fourier coefficients and solving an ordinary differential equation to determine the $r$ dependence. The details are given in Mathews [21].

\subsection{Far-field Green's function}

We now consider the Green's function in the far field $r \gg r_{0}$, which allows us to simplify the result in (14). Using the asymptotic behaviour of the Hankel function gives the Green's function as

$$
G\left(\boldsymbol{x} \mid \boldsymbol{x}_{\mathbf{0}}\right) \sim-\frac{\mathrm{i}}{8 \pi} \sum_{m=0}^{\infty} a_{m} \cos \nu_{m}(\theta+\pi) \cos \nu_{m}\left(\theta_{0}+\pi\right) e^{-\mathrm{i} \pi / 4} e^{-\mathrm{i} \pi m / 4} \mathcal{G}_{m}\left(r \mid r_{0}\right),
$$

where

$$
\mathcal{G}_{m}\left(r \mid r_{0}\right)=\sqrt{\frac{2}{\pi r}} \int_{-\infty}^{\infty} J_{\nu_{m}}\left(\gamma\left(k_{z}\right) r_{0}\right)\left(\gamma\left(k_{z}\right)\right)^{-1 / 2} e^{\mathrm{i} \gamma\left(k_{z}\right) r} e^{\mathrm{i} k_{z}\left(z-z_{0}\right)} \mathrm{d} k_{z}
$$

We then approximate this integral by using the method of stationary phase [2] to give

$$
\mathcal{G}_{m}\left(r \mid r_{0}\right) \sim 2 J_{\nu_{m}}\left(\omega r_{0} \sin \iota\right) \frac{1}{\left|\boldsymbol{x}-\boldsymbol{e}_{\boldsymbol{z}} z_{0}\right|} e^{\mathrm{i} \omega\left|\boldsymbol{x}-\boldsymbol{e}_{\boldsymbol{z}} z_{0}\right|} e^{-\mathrm{i} \pi / 4},
$$

where $\tan \iota=r /\left(z-z_{0}\right)$. Following Howe [14], we assume that $\omega r_{0}$ is small (corresponding to the turbulent sources being close to the aerofoil) and then use the small-argument asymptotic behaviour of the Bessel function

$$
J_{\nu_{m}}\left(\omega r_{0} \sin \iota\right) \sim \frac{\left(\omega r_{0} \sin \iota\right)^{\nu_{m}}}{2^{\nu_{m}} \Gamma\left(\nu_{m}+1\right)},
$$

where $\Gamma$ is the Gamma function. Hence, the terms in the sum in (15) get smaller as $m$ increases so we only need to consider the first few terms, and we only consider the first two (as in Howe [12]), which we denote as $G_{0}$ and $G_{1}$. We calculate that

$$
G_{0}=-\frac{1}{4 \pi\left|\boldsymbol{x}-\boldsymbol{e}_{\boldsymbol{z}} z_{0}\right|} e^{\mathrm{i} \omega\left|\boldsymbol{x}-\boldsymbol{e}_{\boldsymbol{z}} z_{0}\right|} \text { and } G_{1}=-\frac{1}{\pi \sqrt{2 \pi \mathrm{i}}} \frac{\sqrt{\omega} \sqrt{r} \sin (\theta / 2) \sqrt{r_{0}} \sin \left(\theta_{0} / 2\right)}{\left|\boldsymbol{x}-\boldsymbol{e}_{\boldsymbol{z}} z_{0}\right|^{3 / 2}} e^{\mathrm{i} \omega\left|\boldsymbol{x}-\boldsymbol{e}_{\boldsymbol{z}} z_{0}\right|},
$$

which follows from using $\left|\boldsymbol{x}-\boldsymbol{e}_{\boldsymbol{z}} z_{0}\right| \sin \iota=r$. We note that the jump of $G_{0}$, [G $\left.G_{0}\right]$, is zero and hence we approximate the jump in the Green's function as

$$
\left[G\left(\boldsymbol{x},\left(x_{1}, 0, z_{0}\right) ; \omega\right)\right] \sim\left[G_{1}\right]=-\frac{\sqrt{2}}{\pi \sqrt{\pi \mathrm{i}}} \frac{\sqrt{\omega} \sqrt{r} \sin (\theta / 2)}{\left|\boldsymbol{x}-\boldsymbol{e}_{\boldsymbol{z}} z_{0}\right|^{3 / 2}} e^{\mathrm{i} \omega\left|\boldsymbol{x}-\boldsymbol{e}_{\boldsymbol{z}} z_{0}\right|}\left|x_{1}\right|^{1 / 2} .
$$




\subsection{Serrated aerofoil}

For a serrated aerofoil we consider the cylindrical coordinate system introduced in Section 1.2. For the normal derivative of the Green's function, $G^{\mathscr{F}}$, to vanish on the aerofoil, we now require

$$
\frac{\partial G^{\mathscr{F}}}{\partial \theta^{\star}}\left(\theta^{\star}=-\pi\right)=\frac{\partial G^{\mathscr{F}}}{\partial \theta^{\star}}\left(\theta^{\star}=\pi\right)=0 .
$$

In the new, non-orthogonal, coordinate system the Helmholtz equation and the Dirac delta function become significantly more complicated. We calculate that

$$
\delta\left(\boldsymbol{x}-\boldsymbol{x}_{\mathbf{0}}\right)=\frac{1}{r^{\star}-\mathscr{F}^{\prime}\left(z^{\star}\right) \sin \theta^{\star}} \delta\left(r^{\star}-r_{0}^{\star}\right) \delta\left(\theta^{\star}-\theta_{0}^{\star}\right) \delta\left(z^{\star}-z_{0}^{\star}\right),
$$

and

$$
\begin{aligned}
\Delta G^{\mathscr{F}} & =\frac{\partial^{2} G^{\mathscr{F}}}{\partial r^{\star 2}}\left[1+\left(\mathscr{F}^{\prime}\left(z^{\star}\right) \sin \theta^{\star}\right)^{2}\right]+\frac{1}{r^{\star}} \frac{\partial G^{\mathscr{F}}}{\partial r^{\star}}\left[1+\frac{1}{r^{\star}}\left(\mathscr{F}^{\prime}\left(z^{\star}\right) \cos \theta^{\star}\right)^{2}\right]+\frac{1}{r^{\star} 2} \frac{\partial^{2} G^{\mathscr{F}}}{\partial \theta^{\star 2}}\left[1+\left(\mathscr{F}^{\prime}\left(z^{\star}\right) \cos \theta^{\star}\right)^{2}\right] \\
& -\frac{\partial G^{\mathscr{F}}}{\partial \theta^{\star}}\left[\frac{2}{r^{\star 2}}\left(\mathscr{F}^{\prime}\left(z^{\star}\right)\right)^{2} \sin \theta^{\star} \cos \theta^{\star}\right]-\frac{\partial^{2} G^{\mathscr{F}}}{\partial r^{\star} \partial \theta^{\star}}\left[\frac{1}{r^{\star}}\left(\mathscr{F}^{\prime}\left(z^{\star}\right)\right)^{2} \sin \theta^{\star} \cos \theta^{\star}\right]+\frac{\partial^{2} G^{\mathscr{F}}}{\partial z^{\star 2}}
\end{aligned}
$$

which follows from calculating the Jacobian matrix of the coordinate transform.

To make any progress finding the Green's function of the Helmholtz equation we assume that the serrations are shallow (called the slender body approximation in Howe [12]), so $\left|\mathscr{F}^{\prime}(z)\right|$ is small. This allows us to make the approximation

$$
\Delta G^{\mathscr{F}} \approx \frac{\partial^{2} G^{\mathscr{F}}}{\partial r^{\star 2}}+\frac{1}{r^{\star}} \frac{\partial G^{\mathscr{F}}}{\partial r^{\star}}+\frac{1}{r^{\star 2}} \frac{\partial^{2} G^{\mathscr{F}}}{\partial \theta^{\star 2}}+\frac{\partial^{2} G^{\mathscr{F}}}{\partial z^{\star 2}},
$$

and hence the Green's function $G^{\mathscr{F}}$ for the Helmholtz equation is approximated by the solution to

$$
\frac{\partial^{2} G}{\partial r^{\star 2}}+\frac{1}{r^{\star}} \frac{\partial G}{\partial r^{\star}}+\frac{1}{r^{\star 2}} \frac{\partial^{2} G}{\partial \theta^{\star 2}}+\frac{\partial^{2} G}{\partial z^{\star 2}}+\omega^{2} G=\frac{1}{r^{\star}} \delta\left(r^{\star}-r_{0}^{\star}\right) \delta\left(\theta^{\star}-\theta_{0}^{\star}\right) \delta\left(z^{\star}-z_{0}^{\star}\right) .
$$

We solve this in a similar manner to Section 3.1. We find that the exact solution to (25) is given by (14), but with the coordinates replaced by their starred versions. We then use the method of Section 3.2 to calculate the far-field Green's function, which is given by

$$
G^{\mathscr{F}}\left(\boldsymbol{x} \mid \boldsymbol{x}_{\mathbf{0}}\right) \sim-\frac{1}{4 \pi} \sum_{m=0}^{\infty} a_{m} \cos \nu_{m}\left(\theta^{\star}+\pi\right) \cos \nu_{m}\left(\theta_{0}^{\star}+\pi\right) J_{\nu_{m}}\left(\omega r_{0}^{\star} \sin \iota^{\star}\right) \frac{e^{\mathrm{i} \omega\left|\boldsymbol{x}-\boldsymbol{z}_{\boldsymbol{s}}\right|}}{\left|\boldsymbol{x}-\boldsymbol{z}_{\boldsymbol{s}}\right|} e^{-\mathrm{i} \pi m / 4},
$$

where $\tan \iota^{\star}=r^{\star} /\left(z-z_{0}\right)$ and $\boldsymbol{z}_{\boldsymbol{s}}=\left(\mathscr{F}\left(z_{0}\right), 0, z_{0}\right)$, so that $\left|\boldsymbol{x}-\boldsymbol{z}_{\boldsymbol{s}}\right|^{2}=r^{\star 2}+\left(z-z_{0}\right)$. We then again assume that $\omega r_{0}^{\star}$ is small, so we only have to consider the first two terms in the series, and find that $\left[G_{0}^{\mathscr{F}}\right]=0$. Thus, we find the jump in the Green's function is approximately given by

$$
\left[G^{\mathscr{F}}\left(\boldsymbol{x},\left(x_{1}+\mathscr{F}\left(z_{0}\right), 0, z_{0}\right) ; \omega\right)\right] \sim-\frac{\sqrt{2}}{\pi \sqrt{\pi \mathrm{i}}} \frac{\sqrt{\omega} \sqrt{r^{\star}} \sin \left(\theta^{\star} / 2\right)}{\left|\boldsymbol{x}-\boldsymbol{z}_{\boldsymbol{s}}\right|^{3 / 2}} e^{\mathrm{i} \omega\left|\boldsymbol{x}-\boldsymbol{z}_{\boldsymbol{s}}\right|}\left|x_{1}\right|^{1 / 2} .
$$

In Howe [13] it is argued that one can extend the Green's function in (27) to aerofoils with non-shallow serrations, and that using the Green's function in (27) is expected to be an upper bound on the noise from the edge of the aerofoil. However, this relied on his form of the incident pressure, and was not shown rigorously. Thus, when considering results later we will only consider shallow serrations where $\left|\mathscr{F}^{\prime}\left(z_{0}\right)\right|<1$. 


\subsubsection{Comparison to compact Green's function in Howe [12]}

We can compare the Green's functions in (20) and (27) to the compact Green's function in Howe [12] which involves a potential function $\phi^{\star}$. In the far field $(|\boldsymbol{x}| \rightarrow \infty)$ the Green's function without a serration can be written in the form (since the leading order contribution has $\left[G_{0}\right]=0$ )

$$
G(\boldsymbol{x}, \boldsymbol{y} ; \omega)=-\frac{1}{\pi \sqrt{2 \pi \mathrm{i}}} \frac{\sqrt{\omega} \sin (\theta / 2) \phi^{\star}(\boldsymbol{y})}{|\boldsymbol{x}|} e^{\mathrm{i} \omega|\boldsymbol{x}|},
$$

where for a rigid semi-infinite plate $\phi^{\star}(\boldsymbol{y})=\sqrt{r_{0}} \sin \left(\theta_{0} / 2\right)$. This agrees with Howe [12, Equation 19]. In Howe [12] the serrations are then assumed shallow (or the slender wing approximation), which allows an approximate form of $\phi^{\star}$ to be found, given in Howe [12, Equation 20]. The expressions in (27) and in (28) with the approximate form of $\phi^{\star}$ are then essentially both leading order approximations to the far-field Green's functions in a perturbation series about the size of the serration, so will not necessarily agree exactly. Instead of expanding the Laplacian as a perturbation in serration size in (23), we could instead look for better approximations to $\phi^{\star}$, using say the method of matched expansions.

\subsection{Summary of approximations made}

The approximations we used to derive the Green's function were a far-field approximation $\left(r \gg r_{0}\right)$, turbulence sources are close to the aerofoil ( $\omega r_{0}$ is small), and that any serrations are shallow $\left(\left|\mathscr{F}^{\prime}(z)\right|\right.$ is small). Additionally, we assumed the Mach number was small, so that we only had to find the Green's function of the Helmholtz equation, and that the chord of the aerofoil was sufficiently large that we can approximate it by an infinite half plane. These approximations limit the scope of the Green's function and subsequent results about scattered pressure, but we argue that our results are still useful in understanding the effect of serrated aerofoils analytically.

\subsection{Limitations of our model}

If we satisfy all the approximations discussed in the section above, then our model should provide reasonable results for noise estimations. If the approximations are less well satisfied (for instance the serrations are no longer that shallow), then we would generally expect that we get over-prediction of the noise reduction. For instance, Gruber [8, Figure 4.4] showed that Howe's model over-predicts the sound reduction from serrations, for not particularly shallow serrations.

\section{Synthetic turbulence}

In Haeri et al. [10] and Sescu and Hixon [27] the turbulence is modelled as a sum of synthetic eddies, where each eddy has different parameters and can take different "shapes". The total velocity is given as the sum of a base flow $\boldsymbol{u}_{\mathbf{0}}$ and a turbulent velocity perturbation $\boldsymbol{u}$. The turbulent velocity is given as the curl of a vector $\boldsymbol{\Phi}$, so we have

$$
\boldsymbol{u}_{\mathbf{0}}(\boldsymbol{x}, t)=\left(u_{0}, 0,0\right) \text { and } \boldsymbol{u}(\boldsymbol{x}, t)=\boldsymbol{\nabla} \times \boldsymbol{\Phi}(\boldsymbol{x}, t) .
$$

In Haeri et al. [10], $\boldsymbol{\Phi}$ is given as a sum of eddies, with

$$
\boldsymbol{\Phi}(\boldsymbol{x}, t)=\sum_{l=1}^{N_{e}}\left(\Phi_{x, l}(\boldsymbol{x}, t) \boldsymbol{e}_{\boldsymbol{x}}+\Phi_{y, l}(\boldsymbol{x}, t) \boldsymbol{e}_{\boldsymbol{y}}+\Phi_{z, l}(\boldsymbol{x}, t) \boldsymbol{e}_{\boldsymbol{z}}\right),
$$

where $\Phi_{j, l}$ are shape functions with Gaussian or Mexican hat profiles written as functions of

$$
R_{e, l}(\boldsymbol{x}, t)=\left(x-x_{e, l}-u_{0} t\right)^{2}+\left(y-y_{e, l}\right)^{2}+\left(z-z_{e, l}\right)^{2},
$$


where $\boldsymbol{x}_{\boldsymbol{e}, \boldsymbol{l}}=\left(x_{e, l}, y_{e, l}, z_{e, l}\right)$ denotes the source position of the $l$-th eddy. The number of eddies is given by $N_{e}$. We later choose the numerous parameters of the eddy stochastically. To calculate the pressure we use the (full, non-linearised) momentum equation from the Euler equations;

$$
\rho_{0}\left(\frac{\partial \boldsymbol{u}}{\partial t}+\boldsymbol{u}_{\mathbf{0}} \cdot \boldsymbol{\nabla u}\right)+\nabla p=-\rho_{0} \boldsymbol{u} \cdot \boldsymbol{\nabla} \boldsymbol{u}-\rho\left(\frac{\partial \boldsymbol{u}}{\partial t}+\boldsymbol{u}_{\mathbf{0}} \cdot \nabla \boldsymbol{u}+\boldsymbol{u} \cdot \nabla \boldsymbol{u}\right)
$$

where $p$ and $\rho$ are pressure and density perturbations to the constant pressure $p_{0}$ and constant density $\rho_{0}$. Since $\boldsymbol{\Phi}$ is a function of $R_{e, l}$ we necessarily have

$$
\frac{\partial \boldsymbol{u}}{\partial t}+\boldsymbol{u}_{\mathbf{0}} \cdot \boldsymbol{\nabla} \boldsymbol{u}=\mathbf{0}
$$

and hence to leading order $\nabla p=0$, so the eddies do not create linear pressure perturbations. However, to second order we find

$$
\frac{\partial p}{\partial y}=-\rho_{0} \boldsymbol{u} \cdot \nabla v
$$

where $v$ is the $y$ component of velocity. This non-linear form of the pressure perturbation leads to difficulties when we consider more than one eddy, since each eddy interacts with every other eddy.

\section{Turbulence from a single eddy}

We first consider a single eddy with a Gaussian profile, so we assume $\boldsymbol{\Phi}$ is of the form

$$
\boldsymbol{\Phi}(\boldsymbol{x}, t)=A_{1} e^{-B_{1} R_{e}} \boldsymbol{e}_{\boldsymbol{x}}+A_{2} e^{-B_{2} R_{e}} \boldsymbol{e}_{\boldsymbol{y}}+A_{3} e^{-B_{3} R_{e}} \boldsymbol{e}_{\boldsymbol{z}},
$$

with $R_{e}$ given by (31). Since the total incident pressure $p_{i}$ is given by $p_{i}=p+p_{0}$, with $p_{0}$ constant, then $\partial p_{i} / \partial y=\partial p / \partial y$. Inserting the definition of $\boldsymbol{u}=\boldsymbol{\nabla} \times \boldsymbol{\Phi}$ into (34) gives the normal derivative of the pressure in terms of the components of $\mathbf{\Phi}$

$$
\frac{\partial p_{i}}{\partial y}(x, 0, z, t)=-4 \rho_{0}\left[\left(x-x_{e}-u_{0} t\right) \beta_{12} e^{-\gamma_{12} R_{e}}+\beta_{23}\left(z-z_{e}\right) e^{-\gamma_{23} R_{e}}+\beta_{11} y_{e} e^{-\gamma_{11} R_{e}}+\beta_{33} y_{e} e^{-\gamma_{33} R_{e}}\right]
$$

where $\beta_{j l}=A_{j} A_{l} B_{j} B_{l}$ and $\gamma_{j l}=B_{j}+B_{l}$. Next, we insert the definition of the source position $R_{e}$ and make use of the relations

$$
\int_{\mathbb{R}} t^{m} e^{-\gamma(t-\varsigma)^{2}} e^{\mathrm{i} \omega t} \mathrm{~d} t=e^{\mathrm{i} \omega \varsigma} e^{-\omega^{2} / 4 \gamma} \sqrt{\frac{\pi}{\gamma}}\left[\varsigma+\frac{\mathrm{i} \omega}{2 \gamma}\right]^{m}, \quad m=0,1,
$$

where $\varsigma$ is arbitrary, to calculate the inverse Fourier transform of (36). We find that

$$
\begin{array}{r}
\frac{\partial P_{i}}{\partial y}(x, 0, z, \omega)=-\frac{2 \rho_{0}}{u_{0} \sqrt{\pi}}\left[\beta_{23}\left(z-z_{e}\right) \mathbb{E}_{\gamma_{23}}(\boldsymbol{x}, \omega)+\beta_{11} y_{e} \mathbb{E}_{\gamma_{11}}(\boldsymbol{x}, \omega)\right. \\
\left.+\beta_{33} y_{e} \mathbb{E}_{\gamma_{33}}(\boldsymbol{x}, \omega)-\frac{\mathrm{i} \omega}{2 \gamma_{12} u_{0}} \beta_{12} \mathbb{E}_{\gamma_{12}}(\boldsymbol{x}, \omega)\right]
\end{array}
$$

where $\mathbb{E}_{\gamma}$ is the product of exponential terms and is given by

$$
\mathbb{E}_{\gamma}(\boldsymbol{x}, \omega)=\frac{1}{\sqrt{\gamma}} e^{-\gamma\left(z-z_{e}\right)^{2}} e^{-\gamma y_{e}^{2}} e^{\mathrm{i} \omega\left(x-x_{e}\right) / u_{0}} e^{-\omega^{2} /\left(4 \gamma u_{0}^{2}\right)} .
$$


(a)

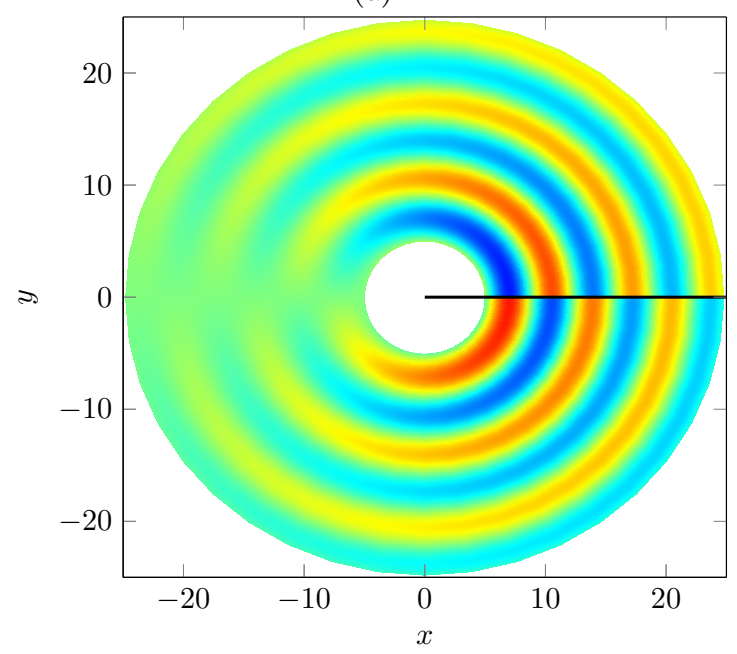

(b)

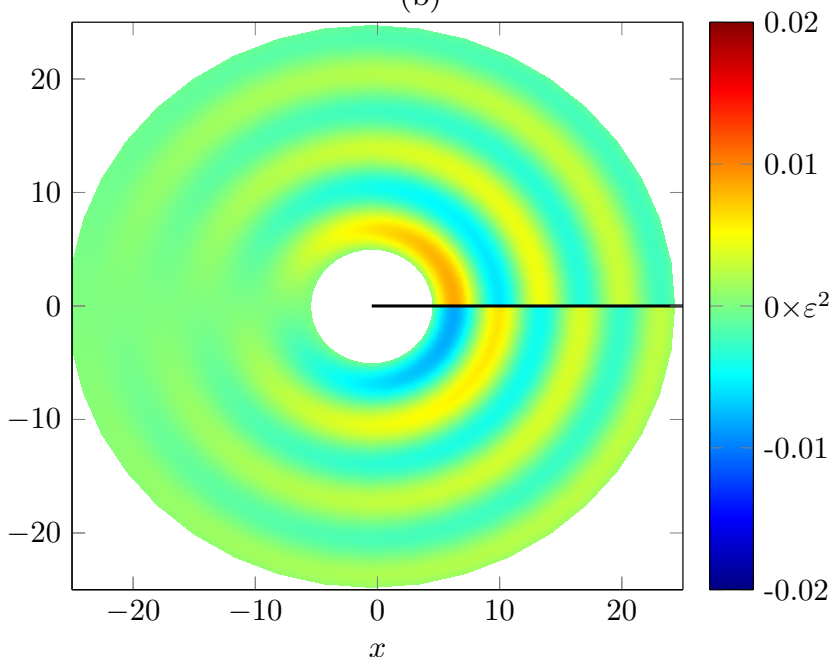

Figure 2: Plot of $\Re\left(P_{s}^{\mathscr{F}}(\boldsymbol{x}, \omega)\right)$ for a straight and serrated edge at fixed $z=-5$. The parameters are $\omega=1, u_{0}=0.25$ with eddy $\boldsymbol{A}=\varepsilon(1,2,1), \boldsymbol{B}=(1,1,2)$ and $x_{e}=(0,0,0)$. (a) $\mathscr{F}(z)=0$ (b) $\mathscr{F}(z)=0.5 \sin (3 z / 2)$.

\subsection{Calculation of scattered pressure}

We now insert the incident pressure from a single eddy in (38) and the Green's function defined in (27) into the Kirchhoff integral in (13) to calculate the scattered pressure. This is given by

$$
P_{s}^{\mathscr{F}}(\boldsymbol{x}, \omega)=A \sqrt{r^{\star}} \sin \left(\frac{\theta^{\star}}{2}\right) \int_{-\infty}^{\infty} \frac{e^{\mathrm{i} \omega\left|\boldsymbol{x}-\boldsymbol{z}_{\boldsymbol{s}}\right|}}{\left|\boldsymbol{x}-\boldsymbol{z}_{\boldsymbol{s}}\right|^{3 / 2}} Q_{1}\left(z_{0}, \omega\right) \mathrm{d} z_{0},
$$

where $A=-2 \rho_{0} \sqrt{2} /\left(\pi^{2} \sqrt{\pi \mathrm{i}}\right)$, and is independent of both eddy and serration, and

$$
Q_{1}\left(z_{0}, \omega\right)=-\frac{\pi \omega^{1 / 2}}{2 \rho_{0}} \int_{0}^{\infty} x_{1}^{1 / 2} \frac{\partial P_{i}}{\partial y}\left(x_{1}+\mathscr{F}\left(z_{0}\right), 0, z_{0}, \omega\right) \mathrm{d} x_{1}
$$

To calculate $Q_{1}$ analytically we use results from Lighthill [18] to calculate integrals such as

$$
\int_{0}^{\infty} x_{1}^{1 / 2} e^{\mathrm{i} \omega x_{1} / u_{0}} \mathrm{~d} x_{1}=\frac{\sqrt{\pi}}{2} \frac{u_{0}^{3 / 2}}{\omega^{3 / 2}}
$$

which we find by multiplying the integrand by $e^{-\varepsilon x_{1}}$ and then taking the limit as $\varepsilon \rightarrow 0$. If we define

$$
Q_{\beta, \gamma}\left(z_{0}, \omega\right)=\frac{u_{0}^{1 / 2}}{2 \omega} \frac{\beta \pi}{\sqrt{\gamma}} e^{3 \pi \mathrm{i} / 4} e^{-\gamma y_{e}^{2}} e^{-\omega^{2} /\left(4 \gamma u_{0}^{2}\right)} e^{-\mathrm{i} \omega x_{e} / u_{0}} e^{-\gamma\left(z_{0}-z_{e}\right)^{2}} e^{\mathrm{i} \omega \mathscr{F}\left(z_{0}\right) / u_{0}}
$$

we conclude that

$$
Q_{1}\left(z_{0}, \omega\right)=\left(z_{0}-z_{e}\right) Q_{\beta_{23}, \gamma_{23}}\left(z_{0}, \omega\right)+y_{e} Q_{\beta_{11}, \gamma_{11}}\left(z_{0}, \omega\right)+y_{e} Q_{\beta_{33}, \gamma_{33}}\left(z_{0}, \omega\right)-\frac{\mathrm{i} \omega}{2 \gamma_{12} u_{0}} Q_{\beta_{12}, \gamma_{12}}\left(z_{0}, \omega\right) .
$$

We then calculate the $z_{0}$ integral in (40), which must be calculated numerically.

\subsection{Initial results}

In Figures 2 and 3 we show some results of our calculations. We perform the integration with respect to $z_{0}$ with a standard numerical solver and plot the real part of $P_{s}^{\mathscr{F}}$ for an eddy with $x_{e}=(0,0,0)$. We set $u_{0}=0.25$, so that the Mach number is small, and additionally set $\omega=1$. In both figures we consider the amplitude $\boldsymbol{A}$ to be multiplied by a scaling factor $\varepsilon$, which ensures that the eddies only cause small 
(a)

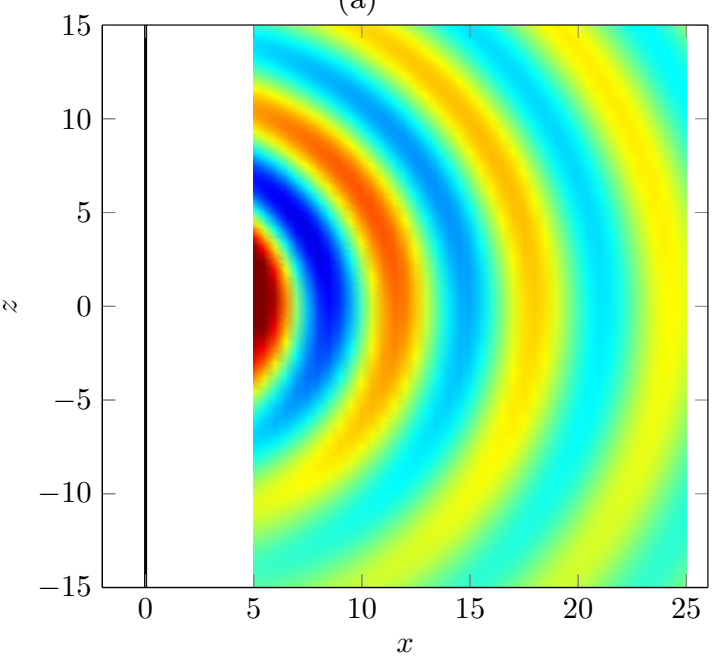

(c)

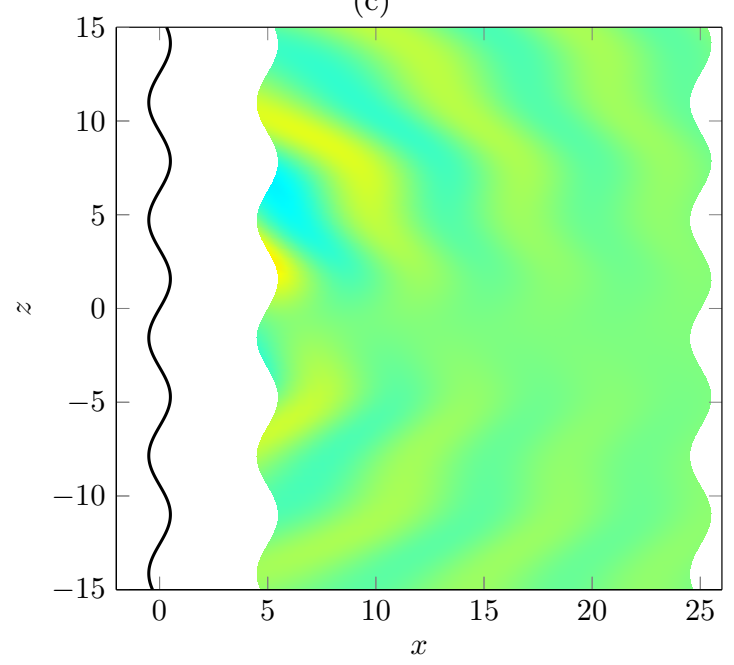

(b)

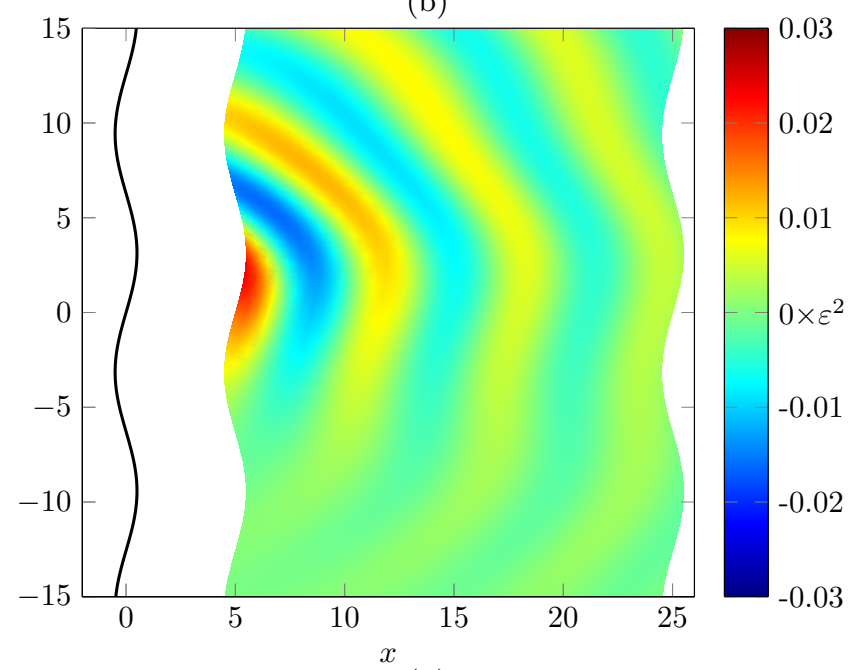

(d)

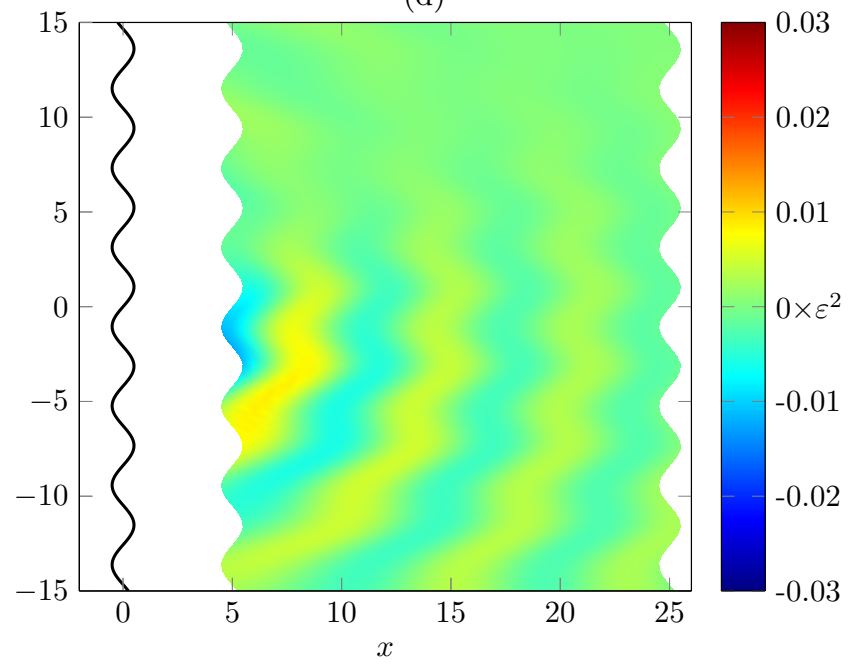

Figure 3: Plot of $\Re\left(P_{s}^{\mathscr{F}}(\boldsymbol{x}, \omega)\right)$ for straight and serrated edges at fixed $\theta^{\star}=\pi$. The parameters are $\omega=1, u_{0}=0.25$ with eddy $\boldsymbol{A}=\varepsilon(1,2,1), \boldsymbol{B}=(1,1,2)$ and $\boldsymbol{x}_{\boldsymbol{e}}=(0,0,0)$. (a) $\mathscr{F}(z)=0, \mathcal{P}^{\mathscr{F}}(\omega)=0.1560 ;(\mathrm{b}) \mathscr{F}(z)=0.5 \sin (z / 2)$, $\mathcal{P}^{\mathscr{F}}(\omega)=0.0467 ;(\mathrm{c}) \mathscr{F}(z)=0.5 \sin (z), \mathcal{P}^{\mathscr{F}}(\omega)=0.0062 ;(\mathrm{d}) \mathscr{F}(z)=0.5 \sin (3 z / 2), \mathcal{P}^{\mathscr{F}}(\omega)=0.0123$

perturbations to the pressure. Since the eddy is close to the aerofoil, we can use the approximate Green's function from earlier, but it is only valid in the far field so we choose to only plot the pressure for $5<r<25$. We plot different views in the figures; a slice in $x-y$ space for Figure 2 and a projection onto the aerofoil in $x-z$ space in Figure 3. From Figure 3 we could easily find the scatted pressure at other angles by multiplying the result by $\sin \left(\theta^{\star} / 2\right)$.

In both figures the results are quite striking and look very hopeful for the effectiveness of serrations. We see that moving from a straight edge to a leading edge serration of $\mathscr{F}(z)=0.5 \sin (3 z / 2)$ reduces the scattered pressure considerably. The largest serration we consider, $\mathscr{F}(z)=0.5 \sin (3 z / 2)$, has $\left|\mathscr{F}^{\prime}(z)\right|<3 / 4$, so is still a shallow serration and our approximate Green's function is still valid. In Figure 3, we see that the optimum leading edge serration to minimise scattered pressure would be close to $\mathscr{F}(z)=0.5 \sin (z)$, with serrations of larger wavelength causing the scattered pressure to increase. 


\subsection{Power and effectiveness of the serrations}

To get a global measure of the effect of a leading edge serration we consider the power of the scattered pressure, given by

$$
\mathcal{P}^{\mathscr{F}}(R, \omega)=\frac{1}{\varepsilon^{4}} \int_{-R}^{R} \int_{-\pi}^{\pi}\left|P_{s}^{\mathscr{F}}\left(R, \theta^{\star}, z^{\star}, \omega\right)\right|^{2} R \mathrm{~d} \theta^{\star} \mathrm{d} z \text { where }|\boldsymbol{A}| \propto \varepsilon
$$

where we have integrated over a cylindrical shell of diameter $2 R$ and length $2 R$. if we assume that the amplitudes are proportional to a small scaling factor $\varepsilon$ (so that the perturbation are small), then we get an $\varepsilon^{4}$ when calculating the power, which is why we have the scaling factor in (45). When we switch back to non-serrated coordinates $(x, y, z)$ this becomes a wavy cylinder, although as $R \rightarrow \infty$ the difference between integrating over a wavy or straight cylinder can be neglected. The $\theta$ integration is easily calculated analytically, while the $z$ integration needs to be performed numerically. We evaluate the power at $R=15$, since this is sufficiently large to get the power in the far-field.

We compute the power of the serrations used in Figure 3, and the results are given in the figure caption. We see a noticeable reduction in the power of the scatted pressure as we reduce the wavelength of the serration, with the minimum power occurring for a leading edge serration close to $\mathscr{F}(z)=0.5 \sin (z)$.

To measure the sound reduction caused by the serrated aerofoil at a particular frequency, we introduce the measure

$$
p_{\text {diff }}(\omega)=\log _{10}\left(\frac{\mathcal{P}^{\mathscr{F}}(\omega)}{\mathcal{P}^{0}(\omega)}\right)
$$

Note that $p_{\text {diff }}$ does not give the sound reduction in terms of decibels, since we would need to sum the power from the scattered pressure with the power from the incident pressure $\mathcal{P}_{i}(\omega)$. Calculating the incident pressure $P_{i}$ is challenging, due to the form of the source term $S_{\text {source }}$ in (7).

\subsection{Effect of the parameters}

There are a significant amount of parameters to consider, with nine parameters for the eddy (amplitudes, Gaussian strengths and source position), two for the serration (wavelength, amplitude), the frequency and the velocity of the base flow. Rather than doing a complete parametric study, we focus only on the effect of several parameters.

\subsubsection{Amplitude}

First, we consider the effect of different amplitudes on the optimum serration. We choose a leading edge serration of $\mathscr{F}_{\mu}(z)=a \sin (\mu z)$, and vary the amplitude $a$. In Figure 4 we see the effect of the different amplitudes on $p_{\text {diff }}$ as we reduce the wavelength (increase $\mu$ ) of the serrations. We only plot the range of wavelengths such that $\left|\mathscr{F}_{\mu}^{\prime}(z)\right|<1$. The trend from the graph is that as we reduce the amplitude of the serration we reduce the effectiveness of the serrations. For serrations with amplitude $a=4$, we reduce $p_{\text {diff }}$

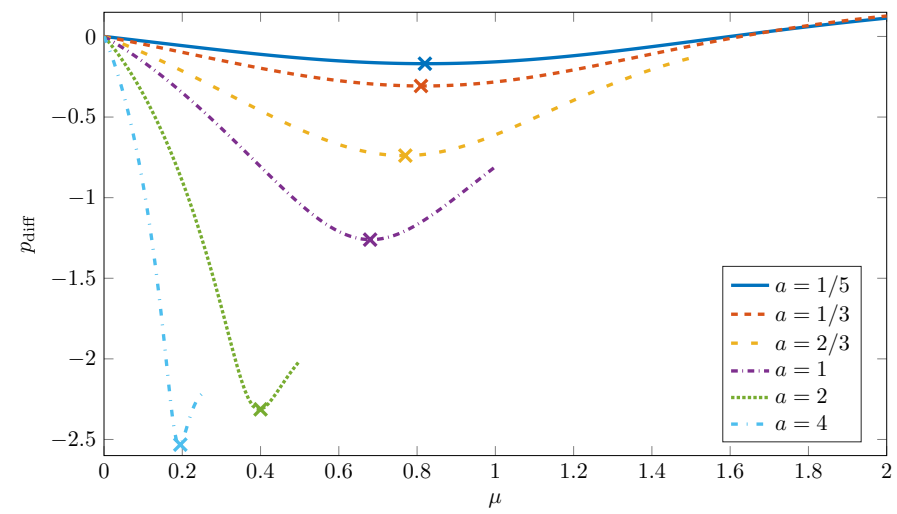

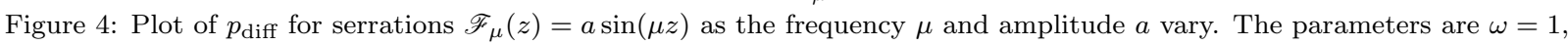
$u_{0}=0.25, \boldsymbol{A}=\varepsilon(-1,1,-2), \boldsymbol{B}=(3,1,2)$ and $\boldsymbol{x}_{\boldsymbol{e}}=(-3,0,1)$. 
(a)

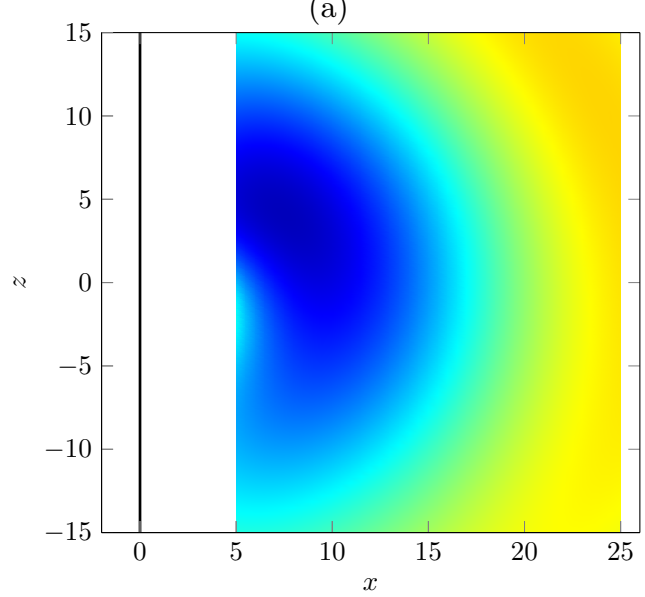

(b)

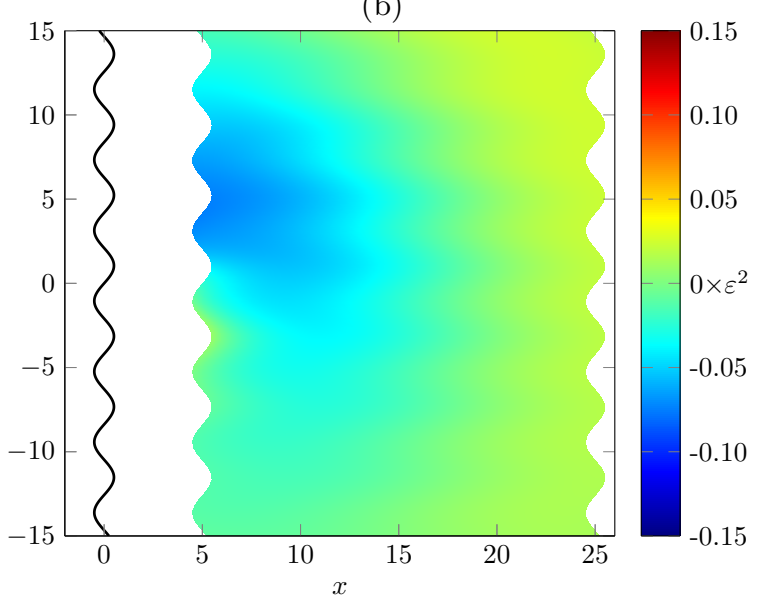

Figure 5: Plot of $\Re\left(P_{s}^{\mathscr{F}}(\boldsymbol{x}, \omega)\right)$ for a straight and serrated edge at fixed $\theta^{*}=\pi$. The parameters are $\omega=0.2, u_{0}=0.25$ with eddy $\boldsymbol{A}=\varepsilon(1,2,1), \boldsymbol{B}=(1,1,2)$ and $\boldsymbol{x}_{\boldsymbol{e}}=(0,0,0)$. (a) $\mathscr{F}(z)=0, \mathcal{P}^{\mathscr{F}}(\omega)=7.7416 ;$ (b) $\mathscr{F}(z)=0.5 \sin (3 z / 2)$, $\mathcal{P}^{\mathscr{F}}(\omega)=1.3418$.

(a)

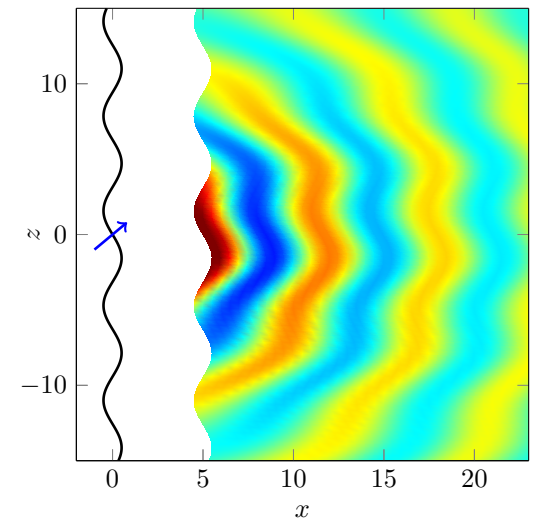

(b)

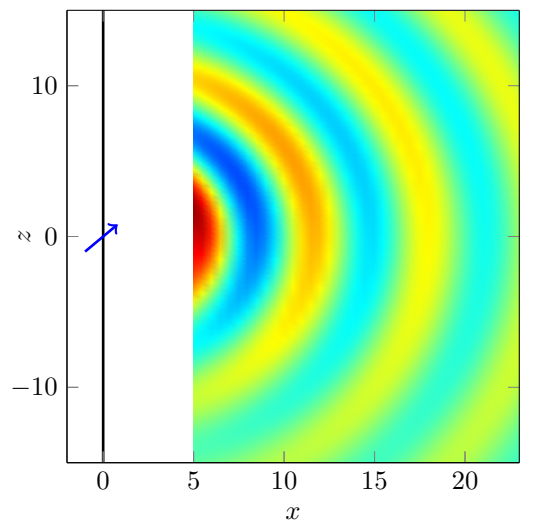

(c)

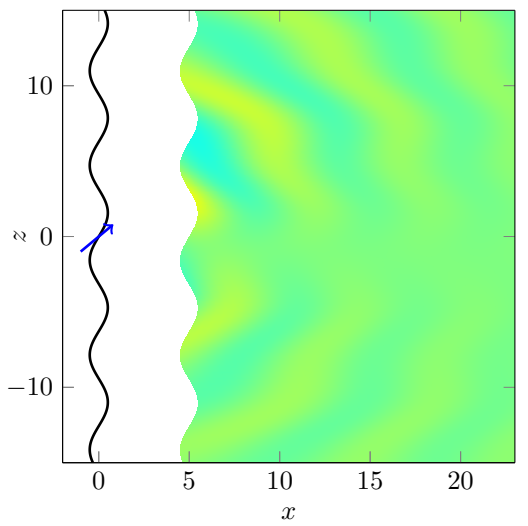

Figure 6: Effect of serrations of the form $\mathscr{F} \mu(z)=0.5 \sin (\mu z)$ on $\Re\left(P_{s}^{\mathscr{F}}(\boldsymbol{x}, \omega)\right)$ for (a) $\mu=-1$; (b) $\mu=0$; (c) $\mu=1$. The parameters are given by $\omega=1, u_{0}=0.25, \boldsymbol{A}=\varepsilon(1,2,1), \boldsymbol{B}=(1,1,2)$ and $\boldsymbol{x}_{\boldsymbol{e}}=(0,0,0)$. The colour scale is the same on each plot.

by around 2.5 with the optimum serration, while when $a=1 / 5$ the maximum reduction in $p_{\text {diff }}$ is only about 0.1. Furthermore, as we reduce the amplitude, the wavelength of the optimum serration (marked with crosses in Figure 4) reduces monotonically to a limit of around 7.5 (corresponding to $\mu=5 / 6$ ).

\subsubsection{Frequency}

We could also consider the effect of the frequency $\omega$ on the effectiveness of the serrations. To calculate the tailored Green's function in Section 3 we assumed that $\omega r_{0}$, the distance from the eddy to the aerofoil multiplied by the frequency, was small, so we only had to take the first two terms in the sum for the Green's function. In Figures 2 and 3 we used $\omega=1$, which is rather large. In Figure 5, we consider $\omega=0.2$, and the rest of the parameters are the same as in Figures 2 and 3. We see that the serrations still reduce the noise by a significant amount.

\subsubsection{Eddy and serration angles}

We next investigate whether a leading edge serration always reduces the noise or whether it can actually increase the noise compared with a straight leading edge. What happens depends on the angle of the eddy in the $x$ - $z$ plane, $\arctan \left(A_{3} / A_{1}\right)$, makes to the serrated leading edge at $z=z_{0}$. In Figure 6 , we plot a straight edge and serrated edges of the form $\mathscr{F}(z)= \pm 0.5 \sin (z)$. We also plot the angle of the eddy as a blue 


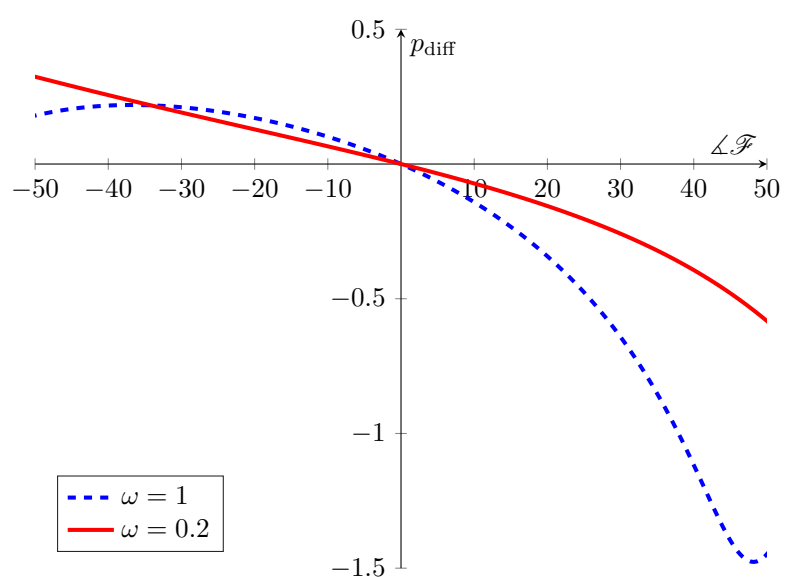

Figure 7: Plot of $p_{\text {diff }}$ against serration angle $\measuredangle \mathscr{F}_{\mu}$ for $\mathscr{F}_{\mu}(z)=0.5 \sin (\mu z)$. The eddy parameters are $\boldsymbol{A}=\varepsilon(1,2,1)$, $\boldsymbol{B}=(1,1,2)$ and $\boldsymbol{x}_{\boldsymbol{e}}=(0,0,0)$. The other parameters are $u_{0}=0.25$ and $\omega=1$ (blue, dashed), $\omega=0.2$ (red, solid).

arrow. In Figure 6c, we see that if the eddy direction and the serration are nearly parallel then we reduce the scattered pressure considerably compared to a straight edge. However, if the eddy and the serration are nearly perpendicular then we increase the noise compared with a straight leading edge, as we see in Figure 6 a.

In Figure 7 we plot $p_{\text {diff }}$ against the angle of the serration (at $z=0$ ) for a serration function $\mathscr{F}_{\mu}(z)=$ $0.5 \sin (\mu z)$. The angle of the serration is given by $\measuredangle \mathscr{F}_{\mu}=\arctan (0.5 \mu)$. From the figure we clearly see that there are serration angles at which we reduce the noise compared to a straight edge but also angles where we increase the noise compared to a straight edge. From Figure 7 we see that the optimum angle (to reduce noise) of the leading edge serration when $\omega=1$ is around $48^{\circ}$, while the worst angle is at around $-37^{\circ}$. When $\omega=0.2$ the optimum angle of the serrations in the range in Figure 7 is $50^{\circ}$ and the worst angle is at $-50^{\circ}$. If we consider a larger range of angles then we find the optimum angle of the serrations is $68^{\circ}$, while the worst serration is at $-68^{\circ}$, neither of which is at all shallow, so it is questionable whether these results are valid. When $\omega=1$ we reduce $p_{\text {diff }}$ by nearly 1.5 with the correct serration and increase it by 0.25 with the worst serration. For $\omega=0.2$ the optimum serration is less effective at reducing noise compared to $\omega=1$, while we increase the noise by more with the worst serration when $\omega=0.2$ compared to $\omega=1$.

\section{Turbulence from multiple eddies}

We now extend the results of Section 5 to first two eddies and then multiple eddies.

\subsection{Two eddies}

Because of the form of the Euler equation in (34) we know that two eddies interact with each other in a non-linear way. We assume both eddies have Gaussian profiles, since this is the case that has been mostly considered in literature. When the eddies have the same source position $R_{e}$, but different amplitudes and Gaussian strengths, we calculate the incident pressure relatively easily. When the eddies have different source positions, the interaction between the two eddies is more complicated, and calculating the incident pressure becomes harder.

We assume the eddies have different source positions so we have two different forms of $R_{e}$. Let us define them as

$$
R_{e}^{\Phi}=\left(x-x_{e}^{\Phi}-u_{0} t\right)^{2}+\left(y-y_{e}^{\Phi}\right)^{2}+\left(z-z_{e}^{\Phi}\right)^{2} \text { and } R_{e}^{\Psi}=\left(x-x_{e}^{\Psi}-u_{0} t\right)^{2}+\left(y-y_{e}^{\Psi}\right)^{2}+\left(z-z_{e}^{\Psi}\right)^{2},
$$

where the eddy associated with $\boldsymbol{\Phi}$ has source position $\left(x_{e}^{\Phi}, y_{e}^{\Phi}, z_{e}^{\Phi}\right)$ and similarly for $\boldsymbol{\Psi}$. We define the displacement between the sources to be

$$
\boldsymbol{d}^{e}=\left(d_{x}^{e}, d_{y}^{e}, d_{z}^{e}\right)=\left(x_{e}^{\Phi}-x_{e}^{\Psi}, y_{e}^{\Phi}-y_{e}^{\Psi}, z_{e}^{\Phi}-z_{e}^{\Psi}\right) .
$$


We calculate that the scattered pressure is of the form

$$
P_{s}^{\mathscr{F}}(\boldsymbol{x}, \omega)=A \sqrt{r^{\star}} \sin \left(\frac{\theta^{\star}}{2}\right) \int_{-\infty}^{\infty} \frac{e^{\mathrm{i} \omega\left|\boldsymbol{x}-\boldsymbol{z}_{s}\right|}}{\left|\boldsymbol{x}-\boldsymbol{z}_{\boldsymbol{s}}\right|^{3 / 2}} Q_{2}^{D}\left(z_{0}, \omega\right) \mathrm{d} z_{0},
$$

where the constant $A$ is the same as in Section 5.1, and is independent of serration and eddy.

To calculate the scattered pressure we use exactly the same method as in Section 5 , and we find that

$$
Q_{2}^{D}\left(z_{0}, \omega\right)=Q_{2}^{D, 1}\left(z_{0}, \omega\right)+2 Q_{2}^{D, 2}\left(z_{0}, \omega\right)
$$

where $Q_{2}^{D, 1}$ and $Q_{2}^{D, 2}$ are given in (A.1) and (A.5) respectively in Appendix A. The integrals needed to calculate $Q_{2}^{D, j}$ in addition to (37) are also given in Appendix A. The term $Q_{2}^{D, 1}$ is due to the linear interaction of the two eddies, while the $Q_{2}^{D, 2}$ term is a correction term due to the eddies having different sources. In the limit $\boldsymbol{d}^{e} \rightarrow 0$ we see that $Q_{2}^{D, 2} \rightarrow 0$, so we get a considerably simpler result.

\subsection{Multiple eddies}

It is clear that we can extend our approach for considering two eddies to $N_{e}$ eddies, since we just need to deal with the cross terms between any two eddies, which consists of expressions similar to $Q_{2}^{D, 2}$ in (A.5). We find that

$$
P_{s}^{\mathscr{F}}(\boldsymbol{x}, \omega)=A \sqrt{r^{\star}} \sin \left(\frac{\theta^{\star}}{2}\right) \int_{-\infty}^{\infty} \frac{e^{\mathrm{i} \omega\left|\boldsymbol{x}-\boldsymbol{z}_{\boldsymbol{s}}\right|}}{\left|\boldsymbol{x}-\boldsymbol{z}_{\boldsymbol{s}}\right|^{3 / 2}} Q_{N_{e}}^{D}\left(z_{0}, \omega\right) \mathrm{d} z_{0},
$$

where

$$
Q_{N_{e}}^{D}\left(z_{0}, \omega\right)=Q_{N_{e}}^{D, 1}\left(z_{0}, \omega\right)+2 Q_{N_{e}}^{D, 2}\left(z_{0}, \omega\right)
$$

For $N_{e}$ eddies, the $Q_{N_{e}}^{D, 1}$ term consists of $N_{e}^{2}$ lines of the form in (A.1), with one line for each eddy $\left(N_{e}\right.$ lines) and $N_{e}\left(N_{e}-1\right)$ lines where the eddies interact with each other. The numbers of terms in the $Q_{N_{e}}^{D, 2}$ term is $N_{e}\left(N_{e}-1\right) / 2$ times the number of terms in (A.5). For stochastic turbulence, we generate the amplitudes $\boldsymbol{\varepsilon} \boldsymbol{A}$, Gaussian distributions $\boldsymbol{B}$ and source positions $\boldsymbol{x}_{\boldsymbol{e}}$ of each eddy stochastically. We choose the simplest possible model, where we use a normal distribution (with zero mean and unit standard deviation) for the amplitudes and source positions, while we choose the Gaussian strengths of the eddies uniformly in $[1,5]$ to avoid them being zero.

In Figure 8 we randomly generate ten eddies, and each of the figures corresponds to a different random seed. The exact eddy parameters are detailed in Appendix B. For each different form of turbulence, we plot how $p_{\text {diff }}$ varies with the serration $\mathscr{F}_{\mu}(z)=0.5 \sin (\mu z)$. We see the effectiveness of the serrations very much depends on the form of the turbulence, and the key to understanding the effect of the serrations is to model the turbulence accurately. For the turbulence in Figures $8 \mathrm{~b}$ and $8 \mathrm{e}$ we see that a leading edge serration can only reduce $p_{\text {diff }}$ by a tiny amount, around 0.1 . In fact, nearly every serration we choose increases the noise compared to a straight leading edge, with $p_{\text {diff }}$ as large as 0.9 in Figure $8 \mathrm{~b}$ and as large as 1.8 in Figure 8 e. In contrast, for the turbulence in Figure 8f, nearly every serration reduces the noise compared to a straight leading edge. We reduce $p_{\text {diff }}$ by 0.8 with the optimum serration in the current model.

In the other figures, we can both increase and decrease the noise by using serrations. In Figure 8a the optimum serration (when $\mu \approx 1$ ) reduces $p_{\text {diff }}$ by around 1 , while the worst serration (when $\mu \approx-0.9$ ) increases $p_{\text {diff }}$ by around 0.4 . In Figure $8 \mathrm{c}$ we reduce $p_{\text {diff }}$ by 0.15 with the optimum serration, but increase it by over 0.2 with the worst serration. In Figure $8 \mathrm{~d}$ we see a reduction in $p_{\text {diff }}$ of about 0.6 when we choose the optimum serration $(\mu \approx 1.7)$, while we increase $p_{\text {diff }}$ by about 0.25 when we choose the worst serration (when $\mu \approx-0.5$ ).

\section{Angle of attack}

Finally, we consider a serrated aerofoil at a small angle of attack $\alpha$. The geometry of the aerofoil is given in Figure 1, and the coordinate system is given in Section 1.2. The scattered pressure $P_{s}$ still satisfies 
(a)

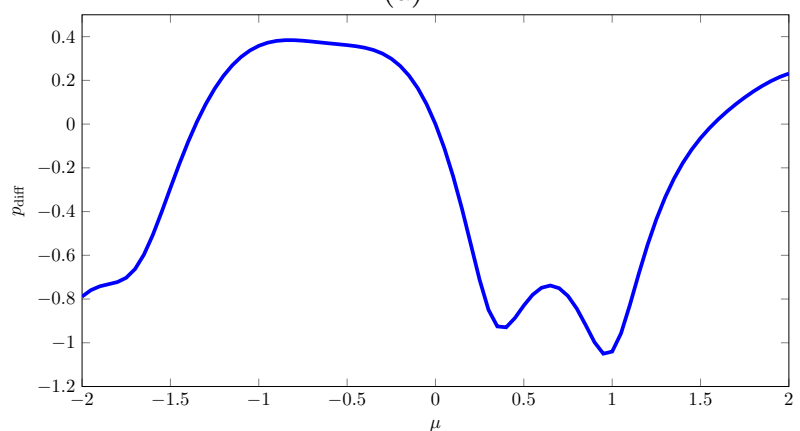

(c)

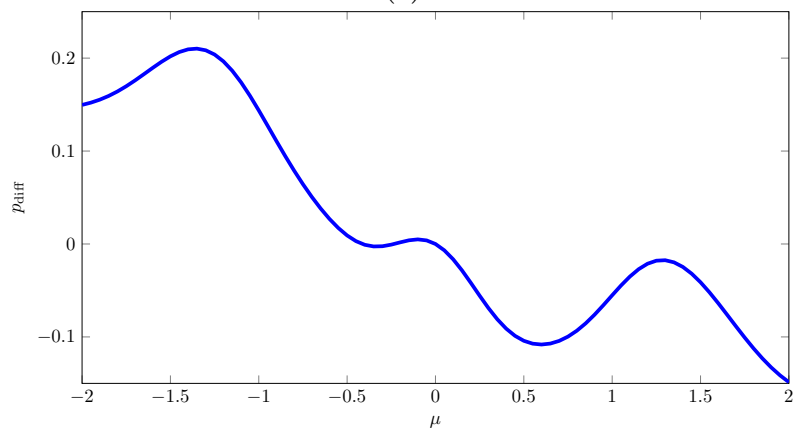

(e)

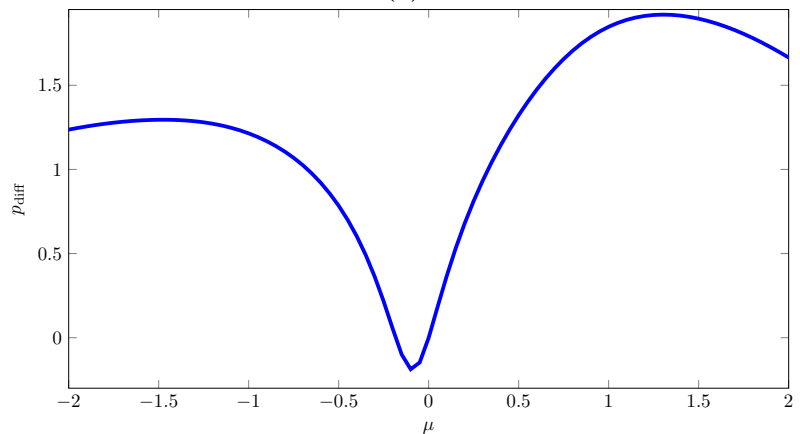

(b)

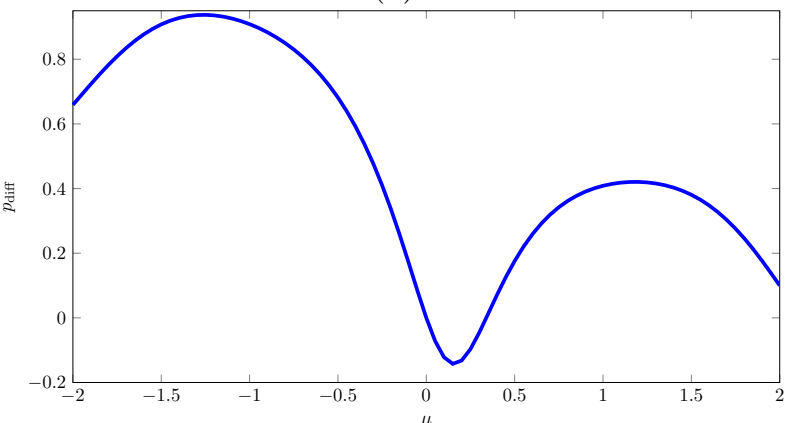

(d)

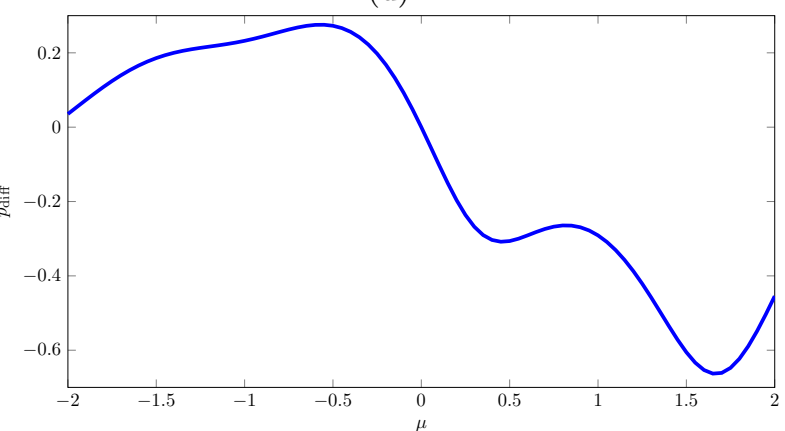

(f)

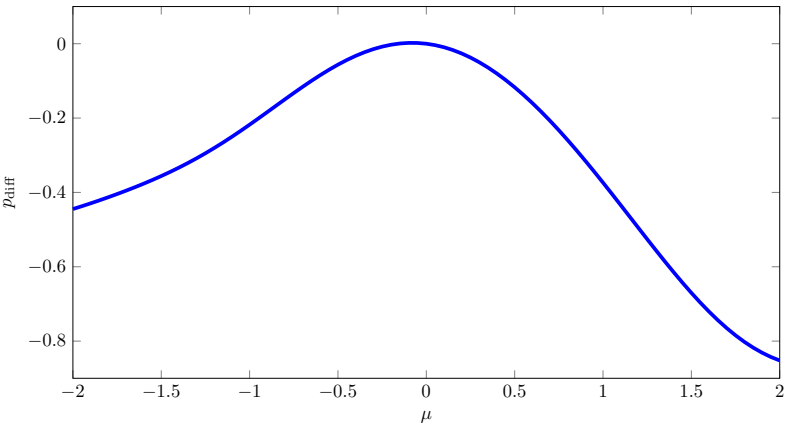

Figure 8: Plot of $p_{\text {diff against serrations } \mathscr{F} \mu}(z)=0.5 \sin (\mu z)$ as $\mu$ varies, for six randomly generated turbulence fields. The eddy parameters are given in Appendix B. The other parameters are $\omega=1$ and $u_{0}=0.25$. Random seeds: (a) Seed 34; (b) Seed 73; (c) Seed 93; (d) Seed 345; (e) Seed 874; (f) Seed 1240.

the Helmholtz equation in (7) since we are assuming a high Reynolds number and low Mach number. We have ignored any effect of the angle of attack on the mean flow, which is still assumed to only be in the $x$ direction. The normal to the aerofoil is now $\boldsymbol{n}=(\sin \alpha, \cos \alpha, 0)$ and hence

$$
\frac{\partial P_{i}}{\partial \boldsymbol{n}}\left(\boldsymbol{x}_{\mathbf{0}}, \omega\right)=\sin \alpha \frac{\partial P_{i}}{\partial x}\left(\boldsymbol{x}_{\mathbf{0}}, \omega\right)+\cos \alpha \frac{\partial P_{i}}{\partial y}\left(\boldsymbol{x}_{\mathbf{0}}, \omega\right) .
$$

Using the Kirchoff integral as in Section 2 , we find that

$$
\left.P_{s}^{\mathscr{F}, \alpha}(\boldsymbol{x}, \omega)=-\int_{-\infty}^{\infty} \int_{0}^{\infty}\left\{\sin \alpha \frac{\partial P_{i}}{\partial x}\left(\boldsymbol{x}_{\mathbf{1}} ; \omega\right)+\cos \alpha \frac{\partial P_{i}}{\partial y}\left(\boldsymbol{x}_{\mathbf{1}} ; \omega\right)\right\}\left[G^{\mathscr{F}, \alpha}\left(\boldsymbol{x}, \boldsymbol{x}_{\mathbf{1}}\right) ; \omega\right)\right] \mathrm{d} x_{1} \mathrm{~d} z_{0},
$$

where

$$
\boldsymbol{x}_{\mathbf{1}}=\left(x_{1}+\mathscr{F}\left(z_{0}\right) \cos \alpha,-x_{1} \tan \alpha-\mathscr{F}\left(z_{0}\right) \sin \alpha, z_{0}\right),
$$

and $\left[G^{\mathscr{F}, \alpha}\right]$ is the jump of the Green's function over the aerofoil at the angle of attack $\alpha$. 


\subsection{Green's function at angle of attack}

For a serrated leading edge, we calculate (using the same method as in Section 3) that the jump of the Green's function is approximately given by

$$
\left[G^{\mathscr{F}, \alpha}\left(\boldsymbol{x}, \boldsymbol{x}_{1} ; \omega\right)\right]=-\frac{2 \sqrt{\omega}}{\pi \sqrt{2 \pi \mathrm{i}}} \sqrt{r^{\star}} \sin \left(\frac{\theta^{\star}}{2}-\frac{\alpha}{2}\right) \frac{e^{\mathrm{i} \omega\left|\boldsymbol{x}-\boldsymbol{z}_{\boldsymbol{s}}\right|}}{\left|\boldsymbol{x}-\boldsymbol{z}_{\boldsymbol{s}}\right|^{3 / 2}}\left(x_{1} \sec \alpha\right)^{1 / 2},
$$

where $\left|\boldsymbol{x}-\boldsymbol{z}_{\boldsymbol{s}}\right|=r^{\star 2}+\left(z-z_{0}\right)^{2}$. Compared to the Green's function at zero angle of attack, the coordinate system has changed, resulting in the $\sin \left(\theta^{\star} / 2\right)$ term being rotated by a factor of $\alpha / 2$ and an additional $\sec ^{1 / 2} \alpha$ term.

\subsection{Synthetic turbulence}

We create the synthetic turbulence using one eddy with a Gaussian profile, in the same way as Section 5. We define the eddy $\boldsymbol{\Phi}$ as in (35) and then the turbulent velocity is given by $\boldsymbol{u}=\boldsymbol{\nabla} \times \boldsymbol{\Phi}$. The mean flow for the incident pressure with no aerofoil present is still given by $\left(u_{0}, 0,0\right)$, and the turbulence is still convected with this mean flow. Using the momentum equation, we calculate

$$
\frac{\partial p_{i}}{\partial x}=-\rho_{0} \boldsymbol{u} \cdot \boldsymbol{\nabla} u \text { and } \frac{\partial p_{i}}{\partial y}=-\rho_{0} \boldsymbol{u} \cdot \boldsymbol{\nabla} v,
$$

in a similar manner to (34). We then substitute in $\boldsymbol{u}=\boldsymbol{\nabla} \times \boldsymbol{\Phi}$, which gives (36) for $\partial p_{i} / \partial y$ and a similar result for $\partial p_{i} / \partial x$.

\subsection{Calculating the scattered pressure}

Using the same method as Section 5 we calculate that

$$
\begin{aligned}
\frac{\partial P_{i}}{\partial y}(x, y, z ; \omega)=\frac{-2 \rho_{0}}{u_{0} \sqrt{\pi}}\left[\beta_{23}\left(z-z_{e}\right) \mathbb{E}_{\gamma_{23}}^{\dagger}(\boldsymbol{x}, \omega)+\beta_{11}\left(y_{e}-y\right)\right. & \mathbb{E}_{\gamma_{11}}^{\dagger}(\boldsymbol{x}, \omega) \\
& \left.+\beta_{33}\left(y_{e}-y\right) \mathbb{E}_{\gamma_{33}}^{\dagger}(\boldsymbol{x}, \omega)-\frac{\mathrm{i} \omega}{2 \gamma_{12} u_{0}} \beta_{12} \mathbb{E}_{\gamma_{12}}^{\dagger}(\boldsymbol{x}, \omega)\right],
\end{aligned}
$$

and

$$
\begin{aligned}
\frac{\partial P_{i}}{\partial x}(x, y, z ; \omega)=\frac{-2 \rho_{0}}{u_{0} \sqrt{\pi}}\left[\beta_{12}\left(y-y_{e}\right) \mathbb{E}_{\gamma_{12}}^{\dagger}(\boldsymbol{x}, \omega)+\beta_{13}\left(z-z_{e}\right)\right. & \mathbb{E}_{\gamma_{13}}^{\dagger}(\boldsymbol{x}, \omega) \\
& \left.+\frac{\mathrm{i} \omega}{2 \gamma_{22} u_{0}} \beta_{22} \mathbb{E}_{\gamma_{22}}^{\dagger}(\boldsymbol{x}, \omega)+\frac{\mathrm{i} \omega}{2 \gamma_{33} u_{0}} \beta_{33} \mathbb{E}_{\gamma_{33}}^{\dagger}(\boldsymbol{x}, \omega)\right]
\end{aligned}
$$

where $\mathbb{E}_{\gamma}^{\dagger}$ is given by

$$
\mathbb{E}_{\gamma}^{\dagger}(\boldsymbol{x}, \omega)=\frac{1}{\sqrt{\gamma}} e^{-\gamma\left(z-z_{e}\right)^{2}} e^{-\gamma\left(y-y_{e}\right)^{2}} e^{\mathrm{i} \omega\left(x-x_{e}\right) / u_{0}} e^{-\omega^{2} /\left(4 \gamma u_{0}^{2}\right)}
$$

Note that $\mathbb{E}_{\gamma}^{\dagger}$ in $(60)$ agrees with $\mathbb{E}_{\gamma}$ in (39) when $y=0$, i.e. at zero angle of attack.

The scattered pressure is then given by

$$
P_{s}^{\mathscr{F}, \alpha}(\boldsymbol{x}, \omega)=A \sqrt{r^{\star}} \sin \left(\frac{\theta^{\star}}{2}-\frac{\alpha}{2}\right) \int_{-\infty}^{\infty} \frac{e^{\mathrm{i} \omega\left|\boldsymbol{x}-\boldsymbol{z}_{s}\right|}}{\left|\boldsymbol{x}-\boldsymbol{z}_{\boldsymbol{s}}\right|^{3 / 2}} Q_{1}^{\alpha}\left(z_{0}, \omega\right) \mathrm{d} z_{0},
$$

where $A$ is the constant from Section 5.1. We find that

$$
Q_{1}^{\alpha}\left(z_{0}, \omega\right)=\sin \alpha Q_{1}^{\alpha, x}\left(z_{0}, \omega\right)+\cos \alpha Q_{1}^{\alpha, y}\left(z_{0}, \omega\right),
$$


where

$$
Q_{1}^{\alpha, j}\left(z_{0}, \omega\right)=-\frac{\pi \omega^{1 / 2}}{2 \rho_{0}} \int_{0}^{\infty} x_{1}^{1 / 2} \frac{\partial P_{i}}{\partial j}\left(x_{1}+\mathscr{F}\left(z_{0}\right) \cos \alpha,-x_{1} \tan \alpha-\mathscr{F}\left(z_{0}\right) \sin \alpha, z_{0}, \omega\right) \mathrm{d} x_{1},
$$

so that $Q_{1}^{0}\left(z_{0}, \omega\right)=Q_{1}\left(z_{0}, \omega\right)$. Defining

$$
Q_{\beta, \gamma}^{\alpha}\left(z_{0}, \omega\right)=\frac{\beta \sqrt{\pi}}{\sqrt{\gamma} u_{0}} e^{-\gamma y_{e}^{2}} e^{-\omega^{2} /\left(4 \gamma u_{0}^{2}\right)} e^{-\mathrm{i} \omega x_{e} / u_{0}} e^{-\gamma\left(z_{0}-z_{e}\right)^{2}} e^{\mathrm{i} \omega \mathscr{F}\left(z_{0}\right) \cos \alpha / u_{0}},
$$

and

$$
I_{\gamma}^{\alpha, l}\left(z_{0}, \omega\right)=\int_{0}^{\infty} x_{1}^{l} e^{\mathrm{i} \omega x_{1} / u_{0}} \exp \left[-\gamma \tan ^{2} \alpha\left(x_{1}+\mathscr{F}\left(z_{0}\right) \cos \alpha+y_{e} \cot \alpha\right)^{2}\right] \mathrm{d} x_{1},
$$

then gives

$$
\begin{aligned}
Q_{1}^{\alpha, x}\left(z_{0}, \omega\right)=\left(z_{0}-z_{e}\right) Q_{\beta_{13}, \gamma_{13}}^{\alpha}\left(z_{0}, \omega\right) I_{\gamma_{13}}^{\alpha, 1 / 2}\left(z_{0}, \omega\right) & +\frac{\mathrm{i} \omega}{2 \gamma_{22} u_{0}} Q_{\beta_{22}, \gamma_{22}}^{\alpha}\left(z_{0}, \omega\right) I_{\gamma_{22}}^{\alpha, 1 / 2}\left(z_{0}, \omega\right) \\
& +\frac{\mathrm{i} \omega}{2 \gamma_{33} u_{0}} Q_{\beta_{33}, \gamma_{33}}^{\alpha}\left(z_{0}, \omega\right) I_{\gamma_{33}}^{\alpha, 1 / 2}\left(z_{0}, \omega\right)-Q_{\beta_{12}, \gamma_{12}}^{\alpha}\left(z_{0}, \omega\right) J_{\gamma_{12}}^{\alpha}\left(z_{0}, \omega\right)
\end{aligned}
$$

and

$$
\begin{aligned}
Q_{1}^{\alpha, y}\left(z_{0}, \omega\right)=\left(z_{0}-z_{e}\right) Q_{\beta_{23}, \gamma_{23}}^{\alpha}\left(z_{0}, \omega\right) I_{\gamma_{23}}^{\alpha, 1 / 2}\left(z_{0}, \omega\right) & -\frac{\mathrm{i} \omega}{2 \gamma_{12} u_{0}} Q_{\beta_{12}, \gamma_{12}}^{\alpha}\left(z_{0}, \omega\right) I_{\gamma_{12}}^{\alpha, 1 / 2}\left(z_{0}, \omega\right) \\
& +Q_{\beta_{11}, \gamma_{11}}^{\alpha}\left(z_{0}, \omega\right) J_{\gamma_{11}}^{\alpha}\left(z_{0}, \omega\right)+Q_{\beta_{33}, \gamma_{33}}^{\alpha}\left(z_{0}, \omega\right) J_{\gamma_{33}}^{\alpha}\left(z_{0}, \omega\right),
\end{aligned}
$$

where

$$
J_{\gamma}^{\alpha}\left(z_{0}, \omega\right)=\left(y_{e}+\mathscr{F}\left(z_{0}\right) \sin \alpha\right) I_{\gamma}^{\alpha, 1 / 2}\left(z_{0}, \omega\right)+I_{\gamma}^{\alpha, 3 / 2}\left(z_{0}, \omega\right) \tan \alpha .
$$

When we have no angle of attack

$$
Q_{\beta, \gamma}^{0} I_{\gamma}^{0,1 / 2}=Q_{\beta, \gamma} \text { and } Q_{\beta, \gamma}^{0} J_{\gamma}^{0}=y_{e} Q_{\beta, \gamma},
$$

where $Q_{\beta, \gamma}$ is given in (43) and hence we get agreement with Section 5. We can calculate the integrals $I_{\gamma}^{\alpha, 1 / 2}$ and $I_{\gamma}^{\alpha, 3 / 2}$ analytically, as detailed in Mathews [21, Appendix B.3].

\subsection{Results}

In Figure 9 we plot the scattered pressure for an aerofoil with a straight leading edge and a serrated leading edge $\mathscr{F}(z)=0.5 \sin (z)$, at angle of attack $\alpha=6^{\circ}$. The eddy parameters are given in Figure 9 . We still see a noticeable reduction in the noise, and our serration still remains shallow (with $\left|\mathscr{F}^{\prime}(z)\right|<1 / 2$ ). By inserting (61) into (45) we calculate $\mathcal{P}^{\mathscr{F}, \alpha}$, the power of the scattered pressure for a serration $\mathscr{F}$ at angle of attack $\alpha$. This is given in the figure caption and is noticeably reduced with the serration.

Finally, in Figure 10 we see how the scattered acoustic power varies across different angle of attacks and different leading edge serrations. We consider angles of attack up to $9^{\circ}$, in $3^{\circ}$ increments. We see several features from Figure 10. First, as we increase the angle of attack, the maximum effectiveness of the serrations generally reduces. This is very much what we expect, since increasing the angle of attack effectively reduces the amplitude of the serrations in the $x$ direction, which reduces the effectiveness of the serrations as we saw in Figure 4. However, the maximum effectiveness of the $3^{\circ}$ aerofoil is actually greater than the maximum effectiveness of a flat aerofoil, showing that a small angle of attack could actually be useful in reducing this noise, although of course other aerofoil noise sources such as trailing edge noise could increase. For different eddy parameters we instead found that maximum effectiveness of the serrations decreased monotonically as we increased the angle of angle, showing there is a complex interaction between the eddy and serrated edge at non-zero angle of attack. 
(a)

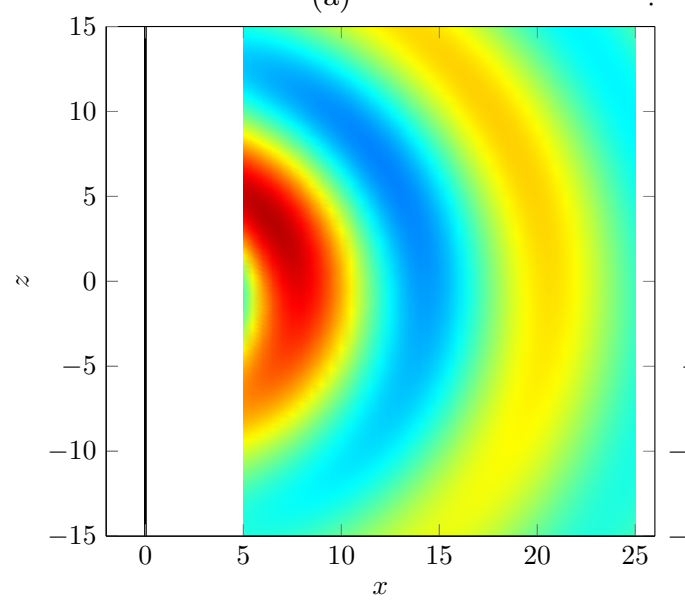

(b)

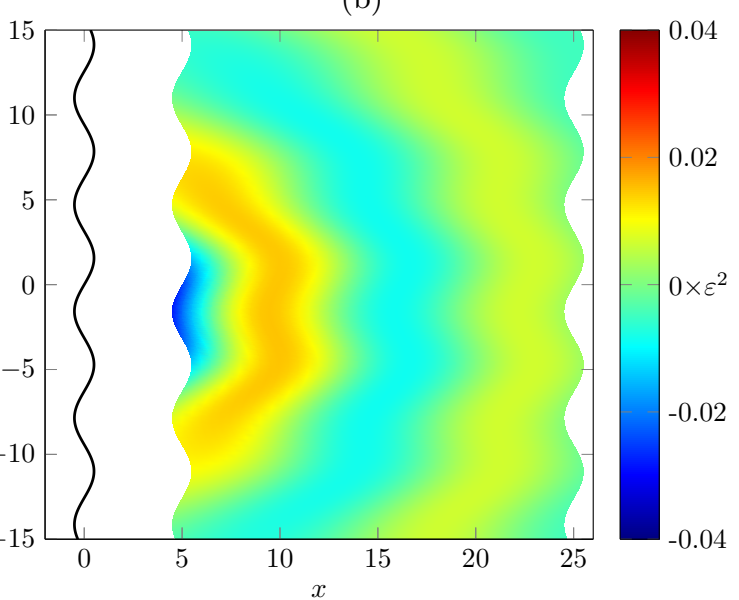

Figure 9: Plot of $\Re\left(P_{s}^{\mathscr{F}, \alpha}(\boldsymbol{x}, \omega)\right)$ for a straight edge and serrated edge at angle of attack $\alpha=6^{\circ}$ and $\theta^{\star}=\pi-\alpha$. The parameters are $\omega=0.5, u_{0}=0.25$ with eddy $\boldsymbol{A}=\varepsilon(1,1,1), \boldsymbol{B}=(1,1,1)$ and $\boldsymbol{x}_{\boldsymbol{e}}=(-1,-0.1,0)$. (a)

$$
\mathscr{F}(z)=0, \mathcal{P}^{\mathscr{F}}, 6(\omega)=0.3071 ;(\mathrm{b}) \mathscr{F}(z)=0.5 \sin (z), \mathcal{P}^{\mathscr{F}}, 6(\omega)=0.1020
$$

The maximum reduction in $p_{\text {diff }}$ is close to 0.8 when the angle of attack is both $0^{\circ}$ and $3^{\circ}$, while when the angle of attack is $9^{\circ}$ the maximum reduction in $p_{\text {diff }}$ is just 0.13 . Second, we see that the optimum serration, where the maximum noise reduction occurs, varies. The wavelength of the optimum serration increases ( $\mu$ decreases) as we increase the angle of attack. Third, we see that as the angle of attack increases the maximum possible value of $p_{\text {diff }}$ increases, from about 0.2 at zero angle of attack to around 0.4 at an angle of attack of $9^{\circ}$.

\section{Conclusion}

In this study we considered the effect of turbulence hitting a single, isolated aerofoil. We analytically calculated the scattered pressure when turbulence represented by synthetic eddies hits the leading edge of a serrated, infinite half plane. To derive this result we made a number of simplifying assumptions, such as assuming the serrations were shallow and that the Mach number was small, and additionally our results are only valid in the far field. This allowed us to calculate the Green's function for Helmholtz's equation on a

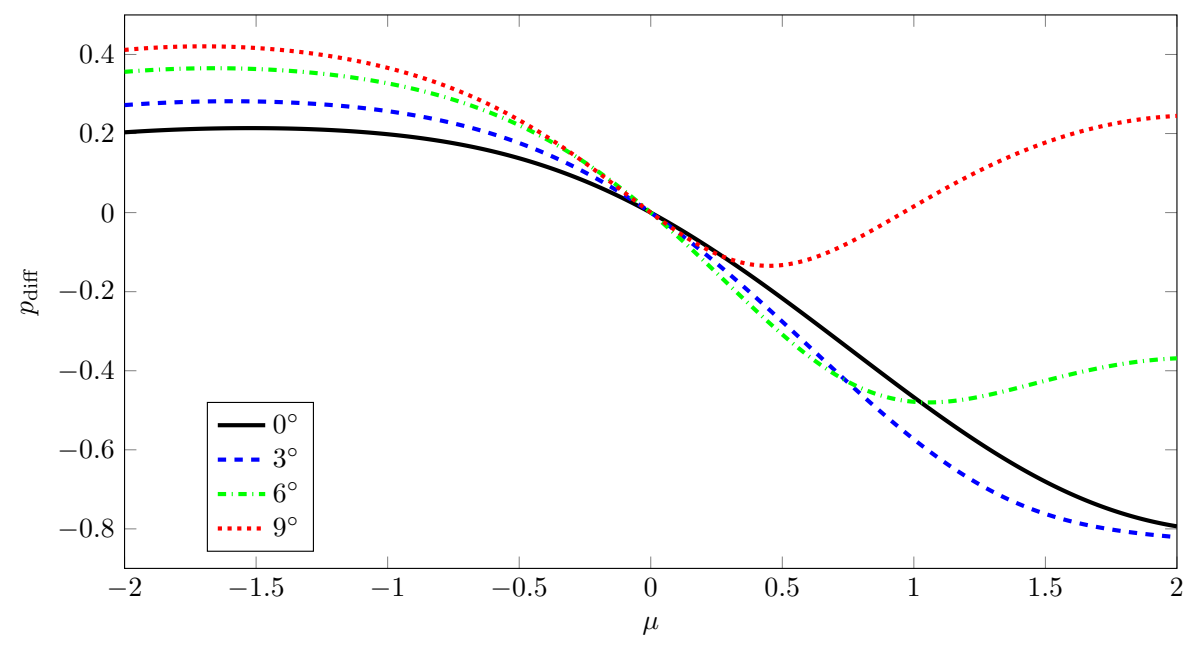

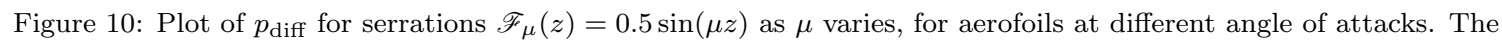
parameters are $\omega=0.5, u_{0}=0.25$ with eddy $\boldsymbol{A}=\varepsilon(1,1,1), \boldsymbol{B}=(1,1,1)$ and $\boldsymbol{x}_{\boldsymbol{e}}=(-1,-0.1,0)$. 
serrated infinite half plane, given by (27). We then considered the cases where we had a single eddy, two eddies or multiples incident eddies, with the Fourier transforms of the scattered pressure given by (40), (49) and (51) respectively.

One of the key results from this paper is Figure 8. This figure shows the level of noise reduction (or increase) as we compare an aerofoil with a serrated leading edge to an aerofoil with a straight leading edge, where we generated the turbulence with ten random synthetic eddies. The figure shows two clear trends. Firstly, there will always be some serrated aerofoil that will reduce the noise compared to a straight leading edge, although the maximum noise reduction depends on the form of the turbulence. Secondly, unless we know the exact form of turbulence, a particular serration could just as easily lead to an increase in the noise as a decrease. We also saw in Figure 10 the effect of the angle of attack, and for aerofoils at higher angles of attack the serration is considerably less effective.

One aspect of future work could be investigated more realistic parameters for the synthetic turbulence, rather than completely random. For example, we could choose the parameters of the eddy so that the von Kármán energy spectrum [29] is achieved. These parameters are given in Haeri et al. [10], Gea-Aguilera et al. [7], and we could use these to generate more realistic turbulence. With a more structured turbulence we would hope that the turbulence affects the effectiveness of the serrations and optimum serration less. Another aspect for future work could be looking at an aerofoil with a finite chord or finite wingspan, but the boundary conditions make it very difficult to find an appropriate tailored Green's function analytically.

One final consideration would be other effects not taken into account by our model. For instance, the serrations could cause separation of the fluid at the leading edge, and this noise source could be just as important as eddies interacting with each other. To date, this effect has only been investigated experimentally, such as in Lacagnina et al. [17].

\section{Acknowledgements}

This work was supported by the UK Engineering and Physical Sciences Research Council (EPSRC) grant EP/H023348/1 for the University of Cambridge Centre for Doctoral Training, the Cambridge Centre for Analysis.

A preliminary version of this work [22] was presented as AIAA paper 2015-2204 at the 21st AIAA/CEAS Aeroacoustics Conference, 22 June - 26 June 2015, Dallas (TX), United States of America.

\section{Appendix A. Analytic calculation of pressure for two eddies with different sources}

The term $Q_{2}^{D, 1}$ is given by

$$
\begin{aligned}
& Q_{2}^{D, 1}\left(z_{0}, \omega\right)=\left(z_{0}-z_{e}^{\Phi}\right) Q_{23}^{\Phi}\left(z_{0}, \omega\right)+y_{e}^{\Phi} Q_{11}^{\Phi}\left(z_{0}, \omega\right)+y_{e}^{\Phi} Q_{33}^{\Phi}\left(z_{0}, \omega\right)-\frac{\mathrm{i} \omega}{2 \gamma_{12}^{B} u_{0}} Q_{12}^{\Phi}\left(z_{0}, \omega\right) \\
& \quad+\left(z_{0}-z_{e}^{\Psi}\right) Q_{23}^{\Psi}\left(z_{0}, \omega\right)+y_{e}^{\Psi} Q_{11}^{\Psi}\left(z_{0}, \omega\right)+y_{e}^{\Psi} Q_{33}^{\Psi}\left(z_{0}, \omega\right)-\frac{\mathrm{i} \omega}{2 \gamma_{12}^{D} u_{0}} Q_{12}^{\Psi}\left(z_{0}, \omega\right) \\
& +\left(z_{0}-z_{e}^{\Psi}\right) Q_{32}^{\Phi, \Psi}\left(z_{0}, \omega\right)+\left(y_{e}^{\Phi}+y_{e}^{\Psi}\right) Q_{11}^{\Phi, \Psi}\left(z_{0}, \omega\right)+\left[-\frac{\mathrm{i} \omega}{2 \gamma_{12}^{B D} u_{0}}+\frac{d_{x}^{e} B_{1}}{\gamma_{12}^{B D}}\right] Q_{12}^{\Phi, \Psi}\left(z_{0}, \omega\right) \\
& \quad+\left(z_{0}-z_{e}^{\Phi}\right) Q_{23}^{\Phi, \Psi}\left(z_{0}, \omega\right)+\left(y_{e}^{\Phi}+y_{e}^{\Psi}\right) Q_{33}^{\Phi, \Psi}\left(z_{0}, \omega\right)+\left[-\frac{\mathrm{i} \omega}{2 \gamma_{21}^{B D} u_{0}}-\frac{d_{x}^{e} D_{1}}{\gamma_{21}^{B D}}\right] Q_{21}^{\Phi, \Psi}\left(z_{0}, \omega\right),
\end{aligned}
$$

where

$$
\begin{aligned}
& Q_{j l}^{\Phi}\left(z_{0}, \omega\right)=\frac{u_{0}^{1 / 2}}{2 \omega} \frac{\beta_{j l}^{A B} \pi e^{3 \pi \mathrm{i} / 4}}{\sqrt{\gamma_{j l}^{B}}} e^{-\gamma_{j l}^{B} y_{e}^{\Phi 2}} e^{-\omega^{2} /\left(4 \gamma_{j l}^{B} u_{0}^{2}\right)} e^{-\mathrm{i} \omega x_{e}^{\Phi} / u_{0}} e^{-\gamma_{j l}^{B}\left(z_{0}-z_{e}^{\Phi}\right)^{2}} e^{\mathrm{i} \omega \mathscr{F}\left(z_{0}\right) / u_{0}}, \\
& Q_{j l}^{\Psi}\left(z_{0}, \omega\right)=\frac{u_{0}^{1 / 2}}{2 \omega} \frac{\beta_{j l}^{C D} \pi e^{3 \pi \mathrm{i} / 4}}{\sqrt{\gamma_{j l}^{D}}} e^{-\gamma_{j l}^{D} y_{e}^{\Psi^{2}}} e^{-\omega^{2} /\left(4 \gamma_{j l}^{D} u_{0}^{2}\right)} e^{-\mathrm{i} \omega x_{e}^{\Psi} / u_{0}} e^{-\gamma_{j l}^{D}\left(z_{0}-z_{e}^{\Psi}\right)^{2}} e^{\mathrm{i} \omega \mathscr{F}\left(z_{0}\right) / u_{0}},
\end{aligned}
$$


and

$$
\begin{aligned}
& Q_{j l}^{\Phi, \Psi}\left(z_{0}, \omega\right)=\frac{u_{0}{ }^{1 / 2}}{2 \omega} \frac{\beta_{j l}^{A D} \pi e^{3 \pi \mathrm{i} / 4}}{\sqrt{\gamma_{j l}^{B D}}} e^{-B_{j} y_{e}^{\Phi 2}-D_{l} y_{e}^{\Psi 2}} e^{-\omega^{2} /\left(4 \gamma_{j l}^{B D} u_{0}^{2}\right)} e^{-B_{j}\left(z_{0}-z_{e}^{\Phi}\right)^{2}-D_{l}\left(z_{0}-z_{e}^{\Psi}\right)^{2}} \\
& \times e^{\mathrm{i} \omega \mathscr{F}\left(z_{0}\right) / u_{0}} \exp \left(\frac{-\mathrm{i} \omega}{u_{0} \gamma_{j l}^{B D}}\left[B_{j} x_{e}^{\Phi}+D_{l} x_{e}^{\Psi}\right]\right) \exp \left(\frac{B_{j} D_{l}}{\gamma_{j l}^{B D}}\left[2 x_{e}^{\Psi} x_{e}^{\Phi}-x_{e}^{\Psi^{2}}-x_{e}^{\Phi^{2}}\right]\right) .
\end{aligned}
$$

The term $Q_{2}^{D, 2}$ is given by the following expression:

$$
\begin{aligned}
& Q_{2}^{D, 2}\left(z_{0}, \omega\right)=B_{1}\left(z_{0}-z_{e}^{\Phi}\right) Q_{13}^{\Phi, \Psi}\left(z_{0}, \omega\right)\left[d_{y}^{e} \frac{\mathrm{i} \omega}{2 \gamma_{13}^{B D} U}-d_{x}^{e} \frac{B_{1} y_{e}^{\Phi}+D_{3} y_{e}^{\Psi}}{\gamma_{13}^{B D}}\right] \\
& -D_{1}\left(z_{0}-z_{e}^{\Psi}\right) Q_{31}^{\Phi, \Psi}\left(z_{0}, \omega\right)\left[d_{y}^{e} \frac{\mathrm{i} \omega}{2 \gamma_{31}^{B D} U}-d_{x}^{e} \frac{B_{3} y_{e}^{\Phi}+D_{1} y_{e}^{\Psi}}{\gamma_{31}^{B D}}\right] \\
& +D_{1}\left(z_{0}-z_{e}^{\Psi}\right) Q_{21}^{\Phi, \Psi}\left(z_{0}, \omega\right)\left[d_{z}^{e} \frac{\mathrm{i} \omega}{2 \gamma_{21}^{B D} U}+d_{x}^{e} z_{0}-d_{x}^{e} \frac{B_{2} z_{e}^{\Phi}+D_{1} z_{e}^{\Psi}}{\gamma_{21}^{B D}}\right] \\
& -B_{1}\left(z_{0}-z_{e}^{\Phi}\right) Q_{12}^{\Phi, \Psi}\left(z_{0}, \omega\right)\left[d_{z}^{e} \frac{\mathrm{i} \omega}{2 \gamma_{12}^{B D} U}+d_{x}^{e} z_{0}-d_{x}^{e} \frac{B_{1} z_{e}^{\Phi}+D_{2} z_{e}^{\Psi}}{\gamma_{12}^{B D}}\right] \\
& +\left(B_{3}-D_{3}\right) Q_{33}^{\Phi, \Psi}\left(z_{0}, \omega\right)\left[\frac{d_{y}^{e}}{2 \gamma_{33}^{B D}}+\frac{\mathrm{i} \omega}{2 \gamma_{33}^{B D} U}\left(d_{y}^{e} \frac{\mathrm{i} \omega}{2 \gamma_{33}^{B D} U}-d_{x}^{e} \frac{B_{3} y_{e}^{\Phi}+D_{3} y_{e}^{\Psi}}{\gamma_{33}^{B D}}\right)\right] \\
& +d_{x}^{e} \frac{2 B_{3} D_{3}}{\gamma_{33}^{B D}} Q_{33}^{\Phi, \Psi}\left(z_{0}, \omega\right)\left(d_{y}^{e} \frac{\mathrm{i} \omega}{2 \gamma_{33}^{B D} U}-d_{x}^{e} \frac{B_{3} y_{e}^{\Phi}+D_{3} y_{e}^{\Psi}}{\gamma_{33}^{B D}}\right) \\
& +D_{3} Q_{23}^{\Phi, \Psi}\left(z_{0}, \omega\right)\left[\frac{d_{z}^{e}}{2 \gamma_{23}^{B D}}+\left(\frac{\mathrm{i} \omega}{2 \gamma_{23}^{B D} U}-d_{x}^{e} \frac{B_{2}}{\gamma_{23}^{B D}}\right)\left(d_{z}^{e} \frac{\mathrm{i} \omega}{2 \gamma_{23}^{B D} U}+d_{x}^{e} z_{0}-d_{x}^{e} \frac{D_{3} z_{e}^{\Phi}+B_{2} z_{e}^{\Psi}}{\gamma_{23}^{B D}}\right)\right] \\
& -B_{3} Q_{32}^{\Phi, \Psi}\left(z_{0}, \omega\right)\left[\frac{d_{z}^{e}}{2 \gamma_{32}^{B D}}+\left(\frac{\mathrm{i} \omega}{2 \gamma_{32}^{B D} U}+d_{x}^{e} \frac{D_{2}}{\gamma_{32}^{B D}}\right)\left(d_{z}^{e} \frac{\mathrm{i} \omega}{2 \gamma_{32}^{B D} U}+d_{x}^{e} z_{0}-d_{x}^{e} \frac{D_{2} z_{e}^{\Phi}+B_{3} z_{e}^{\Psi}}{\gamma_{32}^{B D}}\right)\right] \\
& +\left(B_{1}\left(z_{0}-z_{e}^{\Phi}\right)-D_{1}\left(z_{0}-z_{e}^{\Psi}\right)\right) Q_{11}^{\Phi, \Psi}\left(z_{0}, \omega\right)\left[d_{y}^{e} z_{0}+y_{e}^{\Psi} z_{e}^{\Phi}-y_{e}^{\Phi} z_{e}^{\Psi}\right] \\
& +B_{3} Q_{31}^{\Phi, \Psi}\left(z_{0}, \omega\right)\left[\frac{\mathrm{i} \omega}{2 \gamma_{31}^{B D} U}+d_{x}^{e} \frac{D_{1}}{\gamma_{31}^{B D}}\right]\left[d_{y}^{e} z_{0}+y_{e}^{\Psi} z_{e}^{\Phi}-y_{e}^{\Phi} z_{e}^{\Psi}\right] \\
& -D_{3} Q_{13}^{\Phi, \Psi}\left(z_{0}, \omega\right)\left[\frac{\mathrm{i} \omega}{2 \gamma_{13}^{B D} U}-d_{x}^{e} \frac{B_{1}}{\gamma_{13}^{B D}}\right]\left[d_{y}^{e} z_{0}+y_{e}^{\Psi} z_{e}^{\Phi}-y_{e}^{\Phi} z_{e}^{\Psi}\right] .
\end{aligned}
$$

To derive the equation for $Q_{2}^{D, 1}$ in (A.1) and for $Q_{2}^{D, 2}$ in (A.5) we use the following integrals:

$$
\begin{gathered}
\int_{-\infty}^{\infty} e^{\mathrm{i} \omega t} e^{-\gamma_{1}\left(t-\varsigma_{1}\right)^{2}} e^{-\gamma_{2}\left(t-\varsigma_{2}\right)^{2}}=f_{\omega}\left(\gamma_{1}, \gamma_{2}, \varsigma_{1}, \varsigma_{2}\right), \\
\int_{-\infty}^{\infty} t e^{\mathrm{i} \omega t} e^{-\gamma_{1}\left(t-\varsigma_{1}\right)^{2}} e^{-\gamma_{2}\left(t-\varsigma_{2}\right)^{2}}=\left[\frac{\mathrm{i} \omega}{2 \gamma_{12}}+\frac{\gamma_{1} \varsigma_{1}}{\gamma_{12}}+\frac{\gamma_{2} \varsigma_{2}}{\gamma_{12}}\right] f_{\omega}\left(\gamma_{1}, \gamma_{2}, \varsigma_{1}, \varsigma_{2}\right),
\end{gathered}
$$

and

$$
\int_{-\infty}^{\infty} t^{2} e^{\mathrm{i} \omega t} e^{-\gamma_{1}\left(t-\varsigma_{1}\right)^{2}} e^{-\gamma_{2}\left(t-\varsigma_{2}\right)^{2}}=\left(\frac{1}{2 \gamma_{12}}+\left[\frac{\mathrm{i} \omega}{2 \gamma_{12}}+\frac{\gamma_{1} \varsigma_{1}}{\gamma_{12}}+\frac{\gamma_{2} \varsigma_{2}}{\gamma_{12}}\right]^{2}\right) f_{\omega}\left(\gamma_{1}, \gamma_{2}, \varsigma_{1}, \varsigma_{2}\right),
$$

where $\gamma_{12}=\gamma_{1}+\gamma_{2}$ and

$$
f_{\omega}\left(\gamma_{1}, \gamma_{2}, \varsigma_{1}, \varsigma_{2}\right)=\sqrt{\frac{\pi}{\gamma_{1}+\gamma_{2}}} \exp \left(-\frac{\omega^{2}}{4\left(\gamma_{1}+\gamma_{2}\right)}\right) \exp \left(\mathrm{i} \omega\left[\frac{\gamma_{1} \varsigma_{1}+\gamma_{2} \varsigma_{2}}{\gamma_{1}+\gamma_{2}}\right]\right) \exp \left(\frac{\gamma_{1} \gamma_{2}}{\gamma_{1}+\gamma_{2}}\left[2 \varsigma_{1} \varsigma_{2}-\varsigma_{1}^{2}-\varsigma_{2}^{2}\right]\right) \text {. }
$$

\section{Appendix B. Parameters for stochastic eddies in Section 6}


Table B.1: Parameters for stochastic eddies with seed 34.

\begin{tabular}{|c|c|c|c|c|c|c|c|c|}
\hline$A_{1}$ & $A_{2}$ & $A_{3}$ & $B_{1}$ & $B_{2}$ & $B_{3}$ & $x_{e}$ & $y_{e}$ & $z_{e}$ \\
\hline-0.918 & -1.971 & 0.526 & 1.699 & 2.411 & 1.315 & -0.872 & 2.409 & -0.101 \\
0.789 & 0.686 & -0.129 & 3.059 & 1.434 & 1.137 & 0.324 & -1.523 & 1.496 \\
-1.141 & 1.781 & 0.142 & 4.572 & 4.939 & 3.265 & 1.122 & 2.507 & 1.235 \\
0.385 & 0.294 & -0.319 & 4.695 & 1.604 & 1.599 & -0.663 & -3.034 & -2.116 \\
-0.739 & 0.830 & 1.248 & 4.344 & 2.114 & 1.965 & -0.070 & -0.950 & 0.320 \\
1.792 & 1.767 & -3.276 & 2.999 & 3.797 & 1.134 & -1.241 & 0.483 & -2.203 \\
-0.185 & -0.975 & 1.609 & 4.284 & 3.591 & 3.624 & 2.658 & 1.078 & -1.237 \\
-0.682 & 0.947 & -0.230 & 4.738 & 1.894 & 4.418 & -0.971 & 1.006 & 0.784 \\
-1.357 & -0.592 & -0.180 & 4.179 & 4.207 & 1.799 & 0.956 & 0.106 & 1.612 \\
-0.347 & -0.999 & -0.582 & 2.846 & 3.048 & 4.228 & 1.237 & -0.964 & 1.553 \\
\hline
\end{tabular}

Table B.2: Parameters for stochastic eddies with seed 73

\begin{tabular}{|c|c|c|c|c|c|c|c|c|}
\hline$A_{1}$ & $A_{2}$ & $A_{3}$ & $B_{1}$ & $B_{2}$ & $B_{3}$ & $x_{e}$ & $y_{e}$ & $z_{e}$ \\
\hline 0.344 & -0.327 & 0.767 & 4.280 & 1.326 & 2.063 & 0.322 & -0.550 & -0.174 \\
0.134 & 0.295 & -1.008 & 3.621 & 2.428 & 3.346 & 0.226 & 1.502 & 0.160 \\
0.022 & -1.231 & -1.237 & 1.222 & 3.933 & 2.696 & 1.511 & 0.509 & -0.400 \\
0.346 & 1.000 & 0.108 & 3.757 & 3.925 & 3.539 & -1.572 & -0.631 & -0.446 \\
-0.017 & -1.407 & -1.814 & 3.547 & 1.472 & 1.205 & -0.886 & 0.284 & 0.111 \\
-0.407 & 0.139 & -0.595 & 3.411 & 1.168 & 3.344 & 0.002 & -0.135 & -0.933 \\
-1.212 & -0.686 & -1.676 & 1.486 & 2.275 & 4.856 & 2.149 & 2.044 & 0.457 \\
0.217 & 0.791 & -0.598 & 1.851 & 4.355 & 1.022 & 0.007 & 0.252 & 0.861 \\
1.232 & 0.792 & 0.656 & 3.030 & 3.432 & 3.754 & -0.746 & 0.990 & -0.129 \\
-1.096 & -1.695 & 1.422 & 2.906 & 1.309 & 4.592 & 0.914 & -0.298 & -1.471 \\
\hline
\end{tabular}

Table B.3: Parameters for stochastic eddies with seed 93.

\begin{tabular}{|c|c|c|c|c|c|c|c|c|}
\hline$A_{1}$ & $A_{2}$ & $A_{3}$ & $B_{1}$ & $B_{2}$ & $B_{3}$ & $x_{e}$ & $y_{e}$ & $z_{e}$ \\
\hline 0.358 & 1.653 & 0.169 & 2.910 & 2.863 & 3.882 & -0.718 & 0.664 & 1.255 \\
0.755 & -0.291 & -0.292 & 3.619 & 4.708 & 4.197 & -1.453 & -0.197 & -2.409 \\
-0.402 & -0.094 & 0.354 & 1.804 & 1.510 & 3.571 & -0.007 & -1.340 & 1.352 \\
0.713 & 0.341 & 0.539 & 2.501 & 1.706 & 1.370 & 0.496 & -0.675 & 0.051 \\
0.061 & -0.425 & 1.003 & 2.114 & 2.871 & 2.821 & -0.569 & 0.280 & -0.351 \\
0.503 & -0.793 & -1.928 & 2.299 & 4.786 & 1.821 & 0.918 & -0.087 & -0.591 \\
0.140 & 0.191 & 3.049 & 3.236 & 3.315 & 3.811 & -0.881 & -0.888 & 0.122 \\
0.472 & 0.128 & 0.701 & 4.442 & 1.006 & 1.848 & 2.128 & -2.270 & -1.036 \\
-0.734 & 1.165 & -0.367 & 4.792 & 1.165 & 1.122 & -0.822 & -0.789 & 1.477 \\
-0.409 & -2.033 & -0.031 & 2.819 & 2.688 & 1.138 & 0.522 & 0.475 & 0.251 \\
\hline
\end{tabular}

Table B.4: Parameters for stochastic eddies with seed 345.

\begin{tabular}{|c|c|c|c|c|c|c|c|c|}
\hline$A_{1}$ & $A_{2}$ & $A_{3}$ & $B_{1}$ & $B_{2}$ & $B_{3}$ & $x_{e}$ & $y_{e}$ & $z_{e}$ \\
\hline-0.493 & 0.142 & 1.635 & 2.349 & 1.039 & 2.784 & -0.933 & 0.447 & -1.439 \\
0.381 & -1.997 & -2.156 & 1.855 & 4.165 & 2.312 & -0.251 & 0.430 & -0.477 \\
-0.020 & -0.463 & 0.031 & 4.288 & 4.528 & 4.313 & 0.326 & 0.328 & -1.656 \\
0.480 & 1.805 & -1.038 & 1.678 & 1.750 & 1.463 & 0.287 & 0.435 & -1.500 \\
-0.737 & 0.701 & 1.418 & 4.757 & 4.369 & 1.162 & -2.886 & -0.139 & 0.427 \\
-0.021 & -0.366 & 0.099 & 4.513 & 2.957 & 4.644 & -1.708 & 0.450 & 0.679 \\
0.343 & -1.034 & -0.524 & 1.730 & 2.224 & 2.670 & 0.496 & -0.446 & -0.709 \\
-0.217 & 0.265 & -0.116 & 1.878 & 1.576 & 2.069 & 0.379 & 1.497 & -0.171 \\
-1.973 & -1.270 & -0.720 & 3.638 & 1.527 & 3.786 & -0.796 & -0.007 & 1.431 \\
0.128 & 0.672 & -0.968 & 4.808 & 4.059 & 4.570 & -0.263 & -0.058 & -0.615 \\
\hline
\end{tabular}


Table B.5: Parameters for stochastic eddies with seed 874.

\begin{tabular}{|c|c|c|c|c|c|c|c|c|}
\hline$A_{1}$ & $A_{2}$ & $A_{3}$ & $B_{1}$ & $B_{2}$ & $B_{3}$ & $x_{e}$ & $y_{e}$ & $z_{e}$ \\
\hline 1.424 & -1.175 & -0.678 & 1.671 & 3.550 & 1.900 & 0.454 & -0.333 & 1.035 \\
-0.817 & -0.961 & 0.083 & 2.048 & 2.467 & 4.878 & -0.451 & -0.159 & -0.548 \\
1.216 & -0.146 & 1.495 & 4.922 & 1.110 & 3.206 & -0.922 & -1.026 & 0.268 \\
-0.269 & -0.298 & -0.596 & 4.441 & 4.283 & 4.712 & 0.338 & -0.262 & 1.491 \\
1.895 & 0.020 & -0.114 & 1.757 & 4.630 & 1.325 & 0.143 & -0.578 & 0.431 \\
0.355 & 0.376 & -0.854 & 3.242 & 2.383 & 3.713 & -0.249 & -0.029 & 0.428 \\
0.287 & 1.228 & 1.006 & 4.134 & 2.489 & 3.147 & 0.968 & 0.665 & -0.902 \\
1.033 & 0.804 & -0.048 & 2.370 & 4.421 & 2.243 & -0.874 & -0.607 & 0.267 \\
-1.014 & 0.543 & 0.533 & 4.659 & 2.420 & 2.451 & -0.761 & 0.270 & -0.607 \\
1.099 & -0.708 & -1.681 & 2.293 & 1.341 & 4.357 & 0.081 & 0.747 & 0.085 \\
\hline
\end{tabular}

Table B.6: Parameters for stochastic eddies with seed 1240.

\begin{tabular}{|c|c|c|c|c|c|c|c|c|}
\hline$A_{1}$ & $A_{2}$ & $A_{3}$ & $B_{1}$ & $B_{2}$ & $B_{3}$ & $x_{e}$ & $y_{e}$ & $z_{e}$ \\
\hline 1.352 & -0.052 & 1.096 & 1.989 & 4.459 & 1.122 & -1.097 & 0.817 & 0.403 \\
-1.537 & 0.584 & -1.654 & 4.992 & 2.529 & 3.574 & -1.620 & -0.478 & -0.648 \\
-0.589 & 0.039 & -0.870 & 3.488 & 3.184 & 1.813 & -0.414 & 0.985 & 0.194 \\
1.785 & 1.199 & 0.634 & 3.640 & 2.287 & 3.044 & -0.123 & -0.736 & 0.391 \\
0.501 & 0.500 & -0.882 & 2.278 & 3.315 & 2.569 & 0.796 & 1.590 & -0.936 \\
-1.245 & 0.550 & 1.233 & 2.771 & 4.127 & 4.656 & -0.562 & 0.325 & -0.052 \\
0.115 & 0.100 & -0.553 & 4.450 & 2.757 & 3.238 & -0.671 & -1.507 & 2.038 \\
0.592 & 0.310 & 0.047 & 1.143 & 2.070 & 1.782 & -0.324 & -0.384 & 0.416 \\
0.551 & -0.978 & -0.639 & 3.420 & 4.445 & 4.198 & 0.743 & 1.285 & 0.750 \\
0.385 & -0.854 & 1.334 & 3.162 & 2.808 & 2.677 & -1.344 & -0.336 & -0.827 \\
\hline
\end{tabular}

\section{References}

[1] R. K. Amiet. Noise due to turbulent flow past a trailing edge. Journal of Sound and Vibration, 47(3):387-393, 1976.

[2] C. M. Bender and S. A. Orszag. Advanced mathematical methods for scientists and engineers I: Asymptotic methods and perturbation theory. Springer, 1978.

[3] P Chaitanya, S Narayanan, P Joseph, and J. W. Kim. Leading edge serration geometries for significantly enhanced leading edge noise reductions. In 22nd AIAA/CEAS Aeroacoustics Conference, Lyon, 2016.

[4] P Chaitanya, P. Joseph, S. Narayanan, C. Vanderwel, J. Turner, J. W. Kim, and B. Ganapathisubramani. Performance and mechanism of sinusoidal leading edge serrations for the reduction of turbulence-aerofoil interaction noise. Journal of Fluid Mechanics, 818:435-464, 2017.

[5] A. J. Cooper and N. Peake. Upstream-radiated rotor-stator interaction noise in mean swirling flow. Journal of Fluid Mechanics, 523:219-250, 2005.

[6] Flightpath. Europe's Vision for Aviation: Maintaining Global Leadership \& Serving Society's Needs. http://ec.europa. eu/transport/modes/air/doc/flightpath2050.pdf, 2011. [Online; accessed 03-July-2017].

[7] F. Gea-Aguilera, X. Zhang, X. Chen, J. R. Gill, and T. Nodé-Langlois. Synthetic turbulence methods for leading edge noise predictions. In 21st AIAA/CEAS Aeroacoustics Conference, Dallas (TX), 2015.

[8] M. Gruber. Airfoil noise reduction by edge treatments. PhD thesis, University of Southampton, 2012. URL https: //eprints.soton.ac.uk/349012/.

[9] M. Gruber, P. F. Joseph, and M. Azarpeyvand. An experimental investigation of novel trailing edge geometries on airfoil trailing edge noise reduction. In 19th AIAA/CEAS Aeroacoustic Conference, Berlin, 2013.

[10] S. Haeri, J. W. Kim, S. Narayanan, and P. F. Joseph. 3D calculations of aerofoil-turbulence interaction noise and the effect of wavy leading edges. In 20th AIAA/CEAS Aeroacoustics Conference, Atlanta (GA), 2014.

[11] M. S. Howe. A review of the theory of trailing edge noise. Journal of Sound and Vibration, 61(3):437-465, 1978.

[12] M. S. Howe. Aerodynamic noise of a serrated trailing edge. Journal of Fluids and Structures, 5(1):33-45, 1991.

[13] M. S. Howe. Noise produced by a sawtooth trailing edge. The Journal of the Acoustical Society of America, 90(1):482-487, 1991.

[14] M. S. Howe. Acoustics of fluid-structure interactions. Cambridge University Press, 1998.

[15] M. S. Howe. Trailing edge noise at low Mach numbers. Journal of Sound and Vibration, 225(2):211-238, 1999.

[16] M. S. Howe. Edge-source acoustic Green's function for an airfoil of arbitrary chord, with application to trailing-edge noise. The Quarterly Journal of Mechanics and Applied Mathematics, 54(1):139-155, 2001. 
[17] G. Lacagnina, S. M. Hasheminejad, P. Chaitanya, P. Joseph, T. P. Chong, and O. Stalnov. Leading edge serrations for the reduction of aerofoil separation self-noise. In 23rd AIAA/CEAS Aeroacoustics Conference, Denver (C0), 2017.

[18] M. J. Lighthill. An introduction to Fourier analysis and generalised functions. Cambridge University Press, 1958.

[19] B. Lyu and M. Azarpeyvand. On the noise prediction for serrated leading edges. Journal of Fluid Mechanics, 826:205-234, 2017.

[20] B. Lyu, M. Azarpeyvand, and S. Sinayoko. Prediction of noise from serrated trailing edges. Journal of Fluid Mechanics, 793:556-588, 2016.

[21] J. R. Mathews. Mathematical modelling of noise generation in turbofan aeroengines using Green's functions. PhD thesis, University of Cambridge, 2016. URL https://doi.org/10.17863/CAM.8962.

[22] J. R. Mathews and N. Peake. Noise generation by turbulence interacting with an aerofoil with a serrated leading edge. In 21st AIAA/CEAS Aeroacoustics Conference, Dallas (TX), 2015.

[23] S. Narayanan, P. F. Joseph, S. Haeri, J. W. Kim, C. Paruchuri, and C. Polacsek. Noise reduction studies from the leading edge of serrated flat plates. In 20th AIAA/CEAS Aeroacoustics Conference, Atlanta (GA), 2014.

[24] N. Peake and A. B. Parry. Modern challenges facing turbomachinery aeroacoustics. Annual Review of Fluid Mechanics, 44:227-248, 2012.

[25] S. W. Rienstra and A. Hirschberg. An introduction to acoustics. Eindhoven University of Technology, 2003.

[26] M. Roger and S. Moreau. Back-scattering correction and further extensions of Amiet's trailing-edge noise model. Part 1: theory. Journal of Sound and Vibration, 286(3):477-506, 2005.

[27] A. Sescu and R. Hixon. Toward low-noise synthetic turbulent inflow conditions for aeroacoustic calculations. International Journal for Numerical Methods in Fluids, 73(12):1001-1010, 2013.

[28] J. M. Tyler and T. G. Sofrin. Axial flow compressor noise studies. SAE Technical Paper, 1962.

[29] K. D. Wilson. Turbulence models and the synthesis of random fields for acoustic wave propagation calculations. Technical Report ARL-TR-1677, 1998. 